\title{
3D model of bow shocks
}

\author{
M. Gustafsson ${ }^{1}$, T. Ravkilde ${ }^{2}$, L.E. Kristensen ${ }^{3}$, S. Cabrit ${ }^{4}$, D. Field ${ }^{2}$, and G. Pineau des Forêts ${ }^{4,5}$ \\ 1 Max Planck Institute for Astronomy, Königstuhl 17, 69117 Heidelberg, Germany \\ e-mail: gustafsson@mpia.de \\ 2 Department of Physics and Astronomy, University of Aarhus, 8000 Aarhus C, Denmark \\ 3 Leiden Observatory, Leiden University, PO Box 9513, 2300 RA Leiden, The Netherlands \\ ${ }^{4}$ LERMA, Observatoire de Paris, UMR 8112 of the CNRS, 61 Av. de l'Observatoire, 75014 Paris, France \\ 5 Institut d'Astrophysique Spatiale, UMR 8617 du CNRS, Université de Paris Sud, 91405 Orsay, France
}

Received 24 July 2009 / Accepted 4 January 2010

\section{ABSTRACT}

Context. Shocks produced by outflows from young stars are often observed as bow-shaped structures in which the $\mathrm{H}_{2}$ line strength and morphology are characteristic of the physical and chemical environments and the velocity of the impact.

Aims. We present a 3D model of interstellar bow shocks propagating in a homogeneous molecular medium with a uniform magnetic field. The model enables us to estimate the shock conditions in observed flows. As an example, we show how the model can reproduce rovibrational $\mathrm{H}_{2}$ observations of a bow shock in OMC1.

Methods. The 3D model is constructed by associating a planar shock with every point on a 3D bow skeleton. The planar shocks are modelled with a highly sophisticated chemical reaction network that is essential for predicting accurate shock widths and line emissions. The shock conditions vary along the bow surface and determine the shock type, the local thickness, and brightness of the bow shell. The motion of the cooling gas parallel to the bow surface is also considered. The bow shock can move at an arbitrary inclination to the magnetic field and to the observer, and we model the projected morphology and radial velocity distribution in the plane-of-sky.

Results. The morphology of a bow shock is highly dependent on the orientation of the magnetic field and the inclination of the flow. Bow shocks can appear in many different guises and do not necessarily show a characteristic bow shape. The ratio of the $\mathrm{H}_{2} v=2-1 \mathrm{~S}(1)$ line to the $v=1-0 \mathrm{~S}(1)$ line is variable across the flow and the spatial offset between the peaks of the lines may be used to estimate the inclination of the flow. The radial velocity comes to a maximum behind the apparent apex of the bow shock when the flow is seen at an inclination different from face-on. Under certain circumstances the radial velocity of an expanding bow shock can show the same signatures as a rotating flow. In this case a velocity gradient perpendicular to the outflow direction is a projection effect of an expanding bow shock lighting up asymmetrically because of the orientation of the magnetic field. With the 3D model we reproduce the brightness levels in three $\mathrm{H}_{2}$ lines as well as the shape and size of a chosen bow shock in OMC1. The inferred bow inclination and the orientation and strength of the magnetic field fit into the pattern suggested by independent observations.

Key words. ISM: jets and outflows - ISM: lines and bands - ISM: magnetic fields - ISM: molecules - circumstellar matter shock waves

\section{Introduction}

Bipolar outflows are an integral part of star formation. Outflows from young stars are driven by jets or winds and are frequently the most dramatic and distinct manifestations of a newborn star. Outflows sweep away part of the parent envelope of the star and shock-excite the ambient molecular gas as they propagate from the star. Down the axis of jets, shocks are often observed as bowshaped structures (e.g., Eislöffel et al. 1994; Reipurth \& Bally 2001; Nissen et al. 2007; Davis et al. 2009), which suggests that they form from deflected gas around the leading head of the jet. The shock excitation of the surrounding cloud induces line emission that is characteristic of the physical and chemical environments and the velocity of the impact (Kristensen et al. 2007). The study of bow shocks reveals information in particular on the shock velocity and pre-shock density, as well as on the launching mechanism of the jets and winds from protostars.

In this paper we focus on the molecular hydrogen emission lines in the near-infrared, rather than on Herbig-Haro bow shocks, which may be observed in the visible. Shock-excited gas emits strongly in several rovibrational $\mathrm{H}_{2}$ lines, of which the $v=1-0 \mathrm{~S}(1)$ line at $2.12 \mu \mathrm{m}$ is the strongest. With the advent of integral field spectroscopy, it is now possible to map a molecular hydrogen flow in many emission lines simultaneously. This provides an excellent foundation for detailed modelling. The morphology of a bow shock projected onto the plane of the sky naturally depends on the viewing angle, but also on the orientation of the magnetic field. Also we show here that the line brightness and line ratios can change quite drastically with viewing angle. Thus, in order to model the shocks in detail and extract the underlying physical conditions convincingly, we need a full 3D model that incorporates the effects of the geometry of the system.

There are two different approaches to constructing threedimensional models of shocks. The first is to perform 3D gasdynamic numerical simulations (Raga et al. 2002, 2007; Baek et al. 2009). This approach can treat more of the physics of the dynamical evolution of the shock, but it has been limited so far to single-fluid hydrodynamical or ideal magneto-hydrodynamical simulations (Raga et al. 2002; Suttner et al. 1997). These do not 
allow us to treat continuous (C-type) shocks where ion-neutral decoupling occurs. The second approach is to assume a geometry of the shock and to treat each element on the bow surface as a planar shock, i.e., one assumes that the cooling zone remains thin with respect to the local curvature (Smith 1991; Smith et al. 2003). This approach can treat both C-shocks and discontinuous (J-type) shocks, and it allows a much more refined treatment of the shock chemistry and cooling at the same time, which is essential to obtain accurate line emission.

Here we are concerned with predicting line emission maps of molecular hydrogen in C-type bow shocks. Therefore we use the second approach. We achieve inclusion of the chemistry and coupling to the physics, via for example the degree of ionisation of the gas, by using the multi-fluid 1D model described in Flower \& Pineau des Forêts (2003). This model includes a large chemical reaction network and solves the full set of magnetohydrodynamical equations self-consistently with the chemistry. The main improvements from similar models by Smith et al. (2003) are the addition of non-equilibrium ionisation, dissociation, cooling, the effect of grains on ion-neutral coupling, and the displacement of post-shock gas parallel to the bow surface.

In this paper we present predictions from the 3D model and we show an example of how the model can be used to reproduce observations of a bow shock in Orion. This yields significantly different results from those of 1D and 2D models (Kristensen et al. 2007, 2008). The Orion Molecular Cloud (OMC1) is the closest site of active massive star formation located at a distance of 414 pc (Menten et al. 2007). OMC1 harbours powerful outflows originating from the BN/IRc2 complex. One outflow in the NW-SE direction has given rise to the fast-moving so-called "bullets" (Axon \& Taylor 1984) and the associated "fingers" (Allen \& Burton 1993). These are dissociative shocks observed in [FeII] emission with $\mathrm{H}_{2}$ bow shocks trailing behind. A slower outflow $\left(\sim 18 \mathrm{~km} \mathrm{~s}^{-1}\right)$ is moving in the NE-SW direction (Genzel et al. 1981; Greenhill et al. 1998; Nissen et al. 2007). The morphology of most of the objects in the slower outflow SW of BN is clearly bow shaped (Stolovy et al. 1998; Schultz et al. 1999; Gustafsson et al. 2003; Lacombe et al. 2004; Kristensen et al. 2007; Colgan et al. 2007). We model one of these bow shocks, which has been observed with the ESO-VLT (Gustafsson 2006). The chosen bow shock has previously been modelled by Kristensen et al. (2008) who created a 2D model by combining 1D shock models and estimated the physical properties along the bow shock. They found that the bow shock is propagating in a homogeneous medium and that shock velocities are lower in the wings compared to the apex. The predictions of shock velocity and magnetic field strength agree with observations (Nissen et al. 2007; Norris 1984; Crutcher et al. 1999). However, the 2D model fails to reproduce simultaneously the width of the emission region and the $\mathrm{H}_{2}$ brightness. Motivated by the results of the $2 \mathrm{D}$ modelling and the fact that projection of a 3D bow shell onto the plane of the sky may change both the width of the emission and the general morphology significantly we set out to improve the modelling with the present 3D model.

The organisation of the paper is as follows. In Sect. 2 we describe how the 3D model is constructed. In Sect. 3 we explore the effects on morphology of the shock and molecular hydrogen emission brightness of the individual input parameters such as shock velocity, pre-shock gas density, viewing angle etc.. We present the predictions of the model of the structure of the $\mathrm{H}_{2} v=$ $1-0 \mathrm{~S}(1)$ emission, the $v=2-1 \mathrm{~S}(1) / v=1-0 \mathrm{~S}(1)$ line ratio, the excitation temperature and the radial velocity for a variety of physical conditions covering a range relevant to OMC1. In Sect. 4 we use the 3D model to test whether the underlying shock

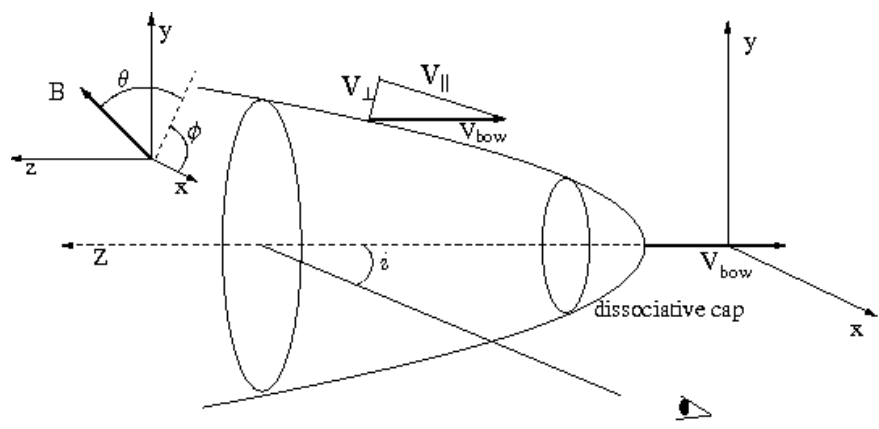

Fig. 1. Geometry of the bow shock model. The bow shock is moving along the $z$-axis at an angle, $i$, to the line of sight which lies in the $z-y$ plane. The direction of a uniform magnetic field is specified by the angles $\theta$ and $\phi$.

conditions can be extracted from observation if only the simpler 1D models are used. A full 3D modelling of the Orion bow shock is performed in Sect. 5. In Sect. 6 we give a summary of our conclusions.

\section{The model}

The method for constructing our 3D model resembles that of Smith (1991) and Smith et al. (2003) in the sense that the $3 \mathrm{D}$ model is built from planar shocks. However, we use a different 1D shock code with a much more extensive chemical network that allows us to follow the non-equilibrium ionisation of gas and grains across the shock, which in turn influence the $\mathrm{C}$ shock thickness and temperature through ion-neutral collisions. The critical velocity for C-shocks also differs between the two models, as we include a more detailed treatment of $\mathrm{H}_{2}$ dissociation and account for the inertia of charged grains. Furthermore, our 3D model includes not only the cooling distance along the shock direction, but also an approximate treatment of the distance travelled by the cooling gas parallel to the bow surface. The geometry and the above improvements are described in more detail in the following sections and in Appendix A.

\subsection{Shock geometry}

We start out by assuming a geometry of the bow shock (Ravkilde 2007). For reasons of simplicity we assume that the shock profile is axisymmetric around the direction of propagation ( $z$-axis in Fig. 1). The shape of the shock profile is parameterised by

$\frac{z}{r_{0}}=\left(\frac{r}{r_{0}}\right)^{\beta}, r=\sqrt{x^{2}+y^{2}}$

where $r_{0}$ determines the "scale of curvature" of the bow, defined as the radius where $z=r$, and $\beta$ determines how "pointed" the shape of the bow profile is. In the following presentation of model predictions we restrict results to $\beta=2.0$ (a paraboloid). However $\beta$ can be varied to match the specific shape of an observed bow shock, as done in Sect. 5.

The shock propagates along the $z$-axis with velocity $v_{\text {bow }}$ into a homogeneous medium with pre-shock density $n_{\mathrm{H}}\left(n_{\mathrm{H}}=\right.$ $\left.n(\mathrm{H})+2 n\left(\mathrm{H}_{2}\right)\right)$ and a uniform magnetic field. The magnetic field strength scales with density as $B=b \sqrt{n_{\mathrm{H}}\left(\mathrm{cm}^{-3}\right)} \mu \mathrm{G}$ where $b$ is the magnetic field scale factor.

We then treat each element on the bow surface as a planar shock with local shock parameters. During the passage of the 
shock wave it is only the velocity component perpendicular to the bow surface, $v_{\perp}$, that contributes to the shock and transforms bulk kinetic energy into thermal energy. Thus, the effective local shock velocity is

$$
v_{\text {shock }}=v_{\perp}=\sin (\omega) v_{\text {bow }}=\frac{v_{\text {bow }}}{r_{0}^{1-\beta} \sqrt{\beta^{2}\left(x^{2}+y^{2}\right)^{\beta-1}+r_{0}^{2 \beta-2}}},
$$

where $\omega=\arctan (\mathrm{d} r / \mathrm{d} z)$ is the angle between the local tangent to the bow surface and the $z$-axis.

The magnetic field acts to cushion the shock and to decouple the ions from the neutral fluid (Draine 1980). We assume that the effective field of oblique shocks is the field component parallel to the shock surface (Smith 1992). Thus $b_{\text {shock }}=b_{\|}$, where $b_{\text {shock }}$ is the local value of the magnetic field scale factor as defined above and

$$
\begin{aligned}
b_{\|}= & \left(b_{x}^{2}+b_{y}^{2}+b_{z}^{2}\right. \\
& \left.-\frac{\left(\beta\left(x^{2}+y^{2}\right)^{\beta / 2-1}\left(x b_{x}+y b_{y}\right)-b_{z} r_{0}^{\beta-1}\right)^{2}}{\beta^{2}\left(x^{2}+y^{2}\right)^{\beta-1}+r_{0}^{2 \beta-2}}\right)^{1 / 2} .
\end{aligned}
$$

\section{2. $1 D$ shock model calculations}

The planar shocks are calculated using the model described in Flower \& Pineau des Forêts (2003). This model involves, as mentioned above, a very detailed treatment of the chemistry and of the atomic and molecular excitation and associated cooling. Chemical events, described by 1065 chemical processes involving 136 species, determine critical parameters such as the degree of ionisation in the medium. Elemental abundances are solar and are distributed among gas phase, grain cores and grain icy mantles following Flower \& Pineau des Forêts (2003). Initial species abundances in the gas phase are derived from a steady state calculation with a standard $\mathrm{H}_{2}$ cosmic ray ionisation rate of $5 \times 10^{-17} \mathrm{~s}^{-1}$ per $\mathrm{H}$ atom. This determines in particular the ratio of atomic to molecular hydrogen and the ionisation fraction in the preshock gas. The PAH abundance is set to $n_{\mathrm{PAH}} / n_{\mathrm{H}}=10^{-6}$. This has important consequences for the magnetosonic speed of the charged fluid and therefore the maximum velocity we can achieve in C-type shocks (Flower \& Pineau des Forêts 2003, see below).

A total of 100 rovibrational level populations of $\mathrm{H}_{2}$ are calculated in parallel with the dynamical and chemical variables, allowing for all radiative transitions and collisional processes which modify level populations. This includes all relevant rotationally and rovibrationally inelastic collisions with $\mathrm{H}, \mathrm{He}$, $\mathrm{H}_{2}$ and electrons and level by level collisional dissociation, as a function of temperature (Le Bourlot et al. 2002). In dissociative shocks $\mathrm{H}_{2}$ is assumed to be reformed with an energy distribution proportional to a Boltzman distribution at $17249 \mathrm{~K}$.

Above a critical shock velocity, $v_{\text {crit }}$, the shock becomes a J-type shock. The critical velocity is defined as the minimum of $v_{\text {dis }}$ and $v_{\text {cms }}$, where $v_{\text {dis }}$ is the velocity at which $\mathrm{H}_{2}$ starts to dissociate (Le Bourlot et al. 2002) and $v_{\mathrm{cms}}$ is the magnetosonic speed of the charged fluid. At shock velocities higher than $v_{\mathrm{cms}}$ the ionic precursor cannot develop ahead of the perturbance (Flower \& Pineau des Forêts 2003). $v_{\mathrm{cms}}$ is approximately equal to the Alfven speed in the charged fluid, $B / \sqrt{4 \pi \rho_{\text {charged }}}$, where $\rho_{\text {charged }}$ is dominated by the grains and is limited by the PAH abundance (Flower \& Pineau des Forêts 2003). $v_{\text {crit }}$ depends on the pre-shock density and magnetic field strength. The greater the component of the magnetic flux perpendicular to the direction of propagation of the shock, the higher is the maximum velocity at which a C-type shock can be sustained. $v_{\text {crit }}$ tends to decrease at higher density owing to the more efficient $\mathrm{H}_{2}$ dissociation.

Given the pre-shock density, $b_{\text {shock}}$, and $v_{\text {shock }}$, we determine for each planar shock whether it is a J-type or a C-type shock. The bow may have a dissociative J-shock cap with oblique Cshocks along the wings as in Fig. 1, but if $\boldsymbol{B}$ is not aligned with the bow axis the minimum of $b_{\|}$will be located somewhere along the wings and the combination of $v_{\perp}$ and $b_{\|}$may result in J-type shocks at that location. Thus, dissociative J-type shocks are not spatially restricted to the apex. Switch-on shocks that might exist when the angle between the shock normal and $\boldsymbol{B}$ approaches zero (Smith 1992; Draine \& McKee 1993), that is $b_{\|} \rightarrow 0$, have not been treated. When $b_{\|}=0$, the planar shock is always a J-type shock in our model.

\subsection{Cooling distance perpendicular and parallel to the bow}

We treat the shock width as resolved when constructing the 3D model. That is, we include the distance travelled by each parcel of gas as the gas cools, which determines the local thickness of the bow shell. This is necessary for C-shocks which are much wider than J-shocks. The shock width is mainly determined by the pre-shock density (see Fig. 8 in Kristensen et al. 2007 ), with the width changing from a few AU at $n_{\mathrm{H}}=10^{7} \mathrm{~cm}^{-3}$ to $\sim 1000 \mathrm{AU}$ at $n_{\mathrm{H}}=10^{4} \mathrm{~cm}^{-3}$. The magnetic field scaling factor, $b$, also has an impact on the shock width although not as drastic. Increasing $b$ and thus the magnetic field naturally increases the shock width by introducing a greater magnetic cushioning effect.

We take into account not only the $1 \mathrm{D}$ cooling distance in the shock direction, but also the distance travelled by the cooling gas parallel to the shock front (in the bow reference frame). In the oblique bow wings, the latter distance is greater than the $1 \mathrm{D}$ cooling distance in the shock direction. This is done in an approximate way by noting that in C-shocks, the bulk of $\mathrm{H}_{2}$ rovibrational emission, which is emitted at temperatures $T>1000 \mathrm{~K}$, occurs at velocities close to $v_{\perp}$, before the gas has had time to slow down by more than a few $\mathrm{km} \mathrm{s}^{-1}$. Thus, to build our emission maps, we assume here that the cooling gas behind each 1D shock is displaced exactly along the $z$-axis until $T \simeq 1000 \mathrm{~K}$. For the purpose of computing centroid velocities we retain the exact velocity vector. We also neglect the transverse pressure gradients and expansion due to the bow curvature, with respect to the compressive term included in the 1D shock models. The accuracy of both approximations is analysed in Appendix A for various B-field strength and preshock densities. Note that this $z$-axis approximation would not be as accurate for pure rotational emission, which is significant to lower $T$.

\subsection{Intensity and centroid velocity maps}

When all the resolved planar shocks are in place in the 3D model we rotate the bow by the inclination angle, $i$, and project it onto the $2 \mathrm{D}$ plane. This yields an image of the bow shock as it would appear in the plane of the sky. In performing this projection we make use of the fact that $\mathrm{H}_{2}$ emission is optically thin for any relevant column densities, since the IR quadrupole rovibrational transitions involved are weak.

The 3D model can produce maps of any of the numerous output parameters from the planar shock simulations. Thus the morphology of shocks can for example be studied by displaying 
the $\mathrm{H}_{2}$ emission or emission from other species. Furthermore we can create excitation diagrams or maps of excitation temperatures by calculating maps from different $\mathrm{H}_{2}$ lines.

We can also predict the centroid velocity of the $\mathrm{H}_{2}$ emission. The pre-shock gas is assumed stationary relative to the observer and it is only $v_{\perp}$ that affects the gas. Hence, in the frame of the observer, the post-shock gas is expanding perpendicular to the bow surface with velocity increasing with distance from the shock front. We construct the radial velocity map by taking the centroid velocity along the line of sight weighted by the local $\mathrm{H}_{2} v=1-0 \mathrm{~S}(1)$ emissivity. We choose radial velocity maps because the radial velocity is the observable quantity which has been reported at 150 mas spatial resolution in Gustafsson et al. (2003) and Nissen et al. (2007).

In building our maps we also need to truncate the bow surface at a maximum outer radius in order to limit the map computing time. We set this maximum outer radius equal to $R_{\text {bow }}=140$ pixels. Both the linear resolution and the radial extent of our maps are then fixed by the elementary pixel size that we adopt. Two methods of choosing the pixel size in the model have been adopted. One approach is to use a specific number of pixels, $n_{\text {thick }}$, to span the thickness of the bow shell, i.e., the maximum cooling distance of the planar shocks along the bow. Using this approach we can fix the ratio of the thickness to truncation radius $R_{\text {bow }}$ of the bow shell, at the expense of letting the size of the bow shock vary. The other approach is to fix the pixel size to a specific value. In this approach $n_{\text {thick }}$ varies freely and is determined by the local shock width. Using this method we fix the map resolution and the maximum size of the bow shock, $R_{\text {bow }}$, but not the relative thickness to radius. In Sect. 3.1 we show examples of both approaches. Otherwise, for the rest of Sect. 3 we have used the second approach and fixed the pixel size to $5.2 \mathrm{AU}$. In all predictions, the radius of curvature $r_{0}$ defined in Eq. (1) is 200 pixels, i.e. $1.4 R_{\text {bow }}$, except in Fig. 4 where it is 71 pixels $=0.5 R_{\text {bow }}$.

\section{Model predictions}

In this section we present predicted bow shock maps by exploring the effects of the individual input parameters on the models. The input parameters can be divided into two groups. The first group, consisting of the pre-shock density, $n_{\mathrm{H}}$, the bow velocity, $v_{\text {bow }}$, and the magnetic field strength, $b$, determines in broad terms the brightness and the thickness of the bow shell. The second group regulates, again broadly, the morphology of the bow shock. This group consists of the orientation of the magnetic field $(\theta, \phi)$ (Fig. 1) which fixes the position of the most strongly emitting shocks on the bow surface and the degree of asymmetry of the map, the angle of inclination, $i$, which influences the line of sight projection, and the geometrical parameters, $r_{0}$ and $\beta$, which determine the curvature of the bow surface and thus how fast the shock conditions vary along the wings (Eqs. (1)-(3)).

The effects of the groups of parameters cannot be entirely separated. It is, however, instructive at first to view them as two separate groups. In reality, the pre-shock density, the bow velocity, and the magnetic field strength will also influence the morphology of the shock since they determine the shell thickness. However the most significant effect on the shape stems from the orientation of the magnetic field and the inclination angle. On the other hand, the orientation of the magnetic field and the inclination angle also influence the brightness due to projection effects, but to a lesser degree than pre-shock density, the bow velocity, and the magnetic field strength, that is, the first group of input parameters.
In the following we first explore the effects on brightness and shell thickness of the first group of input parameters. Then we illustrate how the second group of parameters changes the morphology of the bow shocks. We keep $\beta$ constant at $\beta=2.0$ and give a few examples of the effect of the $r_{0}$ parameter.

Unless otherwise stated, we use the emission from the $\mathrm{H}_{2} v=$ 1-0 S(1) line at $2.1218 \mu \mathrm{m}$ to illustrate the morphology of the projected shocks. This is the strongest NIR rovibrational line of $\mathrm{H}_{2}$ and numerous shocked environments have been mapped in this line with high spatial resolution. In all figures brightnesses are given in $\mathrm{Wm}^{-2} \mathrm{sr}^{-1}$ and sizes in $\mathrm{AU}$.

\subsection{Density}

We start out by investigating the effect of the pre-shock density on shock size and appearance in Fig. 2. The pre-shock density is varied between $10^{4}-10^{6} \mathrm{~cm}^{-3}$ while the other input parameters are kept constant at $v_{\text {bow }}=40 \mathrm{~km} \mathrm{~s}^{-1}, b=5, \beta=2.0$. The input parameters are chosen to resemble the physical conditions of OMC1 as found by Kristensen et al. (2008) by 2D shock modelling. The figure consists of two parts. In the left-hand column we fix the pixel size to $1 / 100$ of the maximum cooling distance of the associated planar shocks so that our bow truncation radius is 1.4 times the cooling length. The relative thickness to size of the bow is thus the same in all three models, and the thickness is also small compared to the radius of curvature $r_{0}$ taken here as $1.4 R_{\text {bow }}$ Since the shock width decreases with increasing density (see also Kristensen et al. 2007) the pixel size and the size of the bow shocks are determined by the pre-shock density. The pixel size decreases from $142 \mathrm{AU}$ at $n_{\mathrm{H}}=10^{4} \mathrm{~cm}^{-3}$, to $27.5 \mathrm{AU}$ at $n_{\mathrm{H}}=10^{5} \mathrm{~cm}^{-3}$ and to $5.2 \mathrm{AU}$ in the $n_{\mathrm{H}}=10^{6} \mathrm{~cm}^{-3}$ model. The bow truncation radius is 140 times larger. The peak brightness in the bow shock increases with increasing density and the emission in the wings becomes relatively stronger at high densities compared to the apex brightness. This arises because a change in the shock velocity in high density systems has a smaller effect on the brightness of the $v=1-0 \mathrm{~S}(1)$ line than at low densities. At high densities, lower $J$ and $v$ levels may become more nearly thermalized and increasing the shock velocity leads to population spread among a greater range of levels (Kristensen et al. 2007).

In the right-hand column of Fig. 2 we have fixed the pixel size to $41.5 \mathrm{AU}$ and thus fixed the outer truncation radius and the curvature radius of the bow shock to the same values of $R_{\text {bow }}=$ $5900 \mathrm{AU}$ and $r_{0}=8300 \mathrm{AU}$, respectively, in all three models. Thus, the relative thickness to radius of the bow shell varies due to variations of shock width. At $n_{\mathrm{H}}=10^{4} \mathrm{~cm}^{-3}$ the shock width is very large compared to the radius of curvature and the bow structure is hardly visible. At $n_{\mathrm{H}}=10^{5} \mathrm{~cm}^{-3}$ the bow shell is thin and the bow structure is clear. And at $n_{\mathrm{H}}=10^{6} \mathrm{~cm}^{-3}$ the shock width is so small that it is barely resolved using the pixel size adopted. From Fig. 2 it is clear that the appearance of a bow shock changes dramatically if the ratio of the shock width to bow radius changes.

In observational data the pre-shock density can be estimated by studying the width of the emitting region as well as the brightness. This has been discussed at length in Kristensen et al. (2008) in which, in 2D models, the observed shock width was found to be a valuable constraint on the physical conditions. 

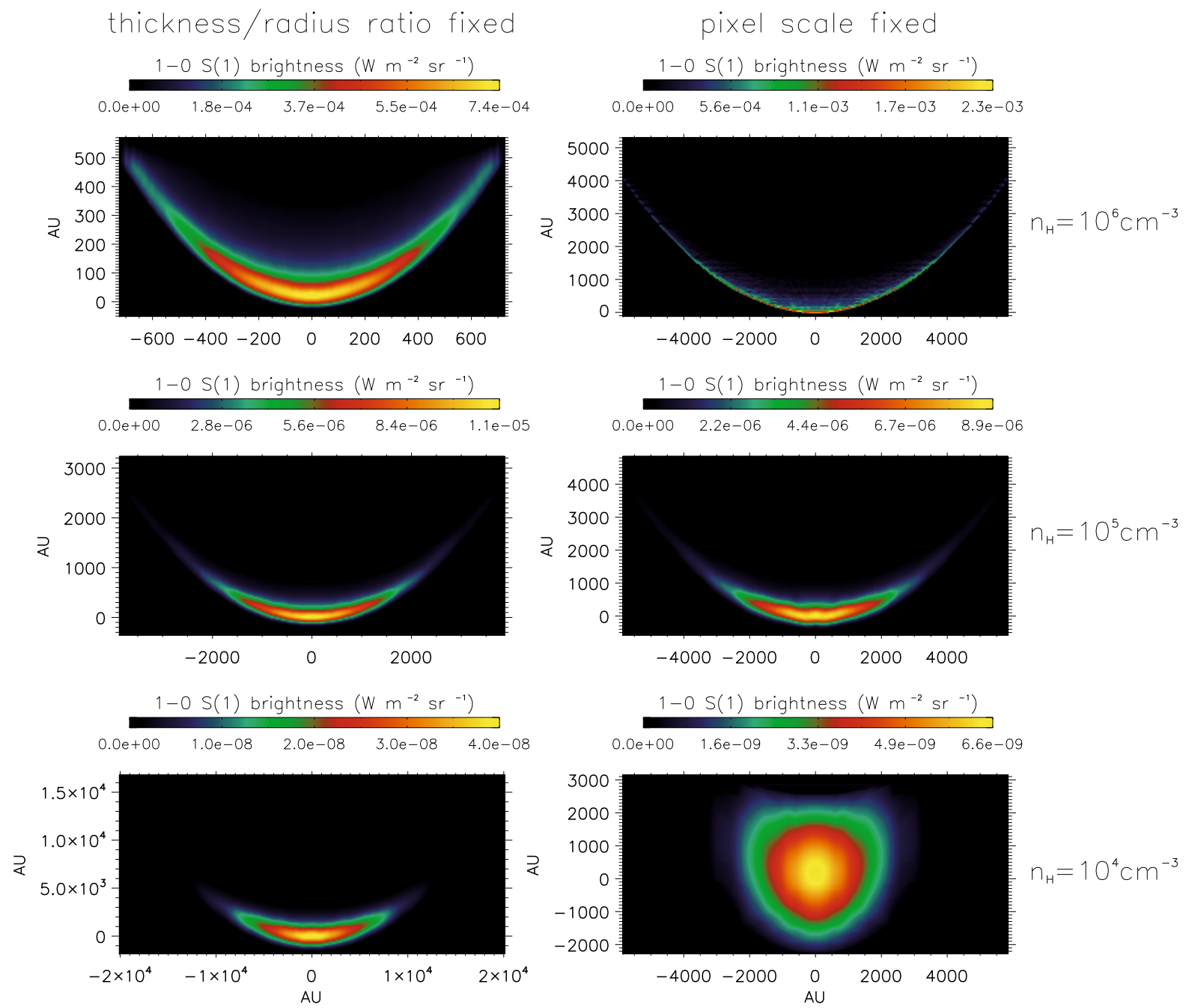

Fig. 2. Brightness in $\mathrm{Wm}^{-2} \mathrm{sr}^{-1}$, size and thickness in $\mathrm{AU}$ of a series of shocks as a function of pre-shock density. From top to bottom, the pre-shock density is $10^{6}, 10^{5}, 10^{4} \mathrm{~cm}^{-3}$. The shock is seen edge-on, $i=90^{\circ}$, with the apex at $(0,0)$. The magnetic field is perpendicular to the flow direction and in the plane of the sky $(\theta=0, \phi=0)$. Other constant input parameters are $v_{\text {bow }}=40 \mathrm{~km} \mathrm{~s}^{-1}, b=5, \beta=2.0, r_{0}=200$ pixels. The colour scale is linear. Left column: the ratio of shell thickness to pixel size is fixed. Therefore the truncation radius $R_{\text {bow }}$ varies proportionally and the ratio of thickness to radius is the same in all three models. Right column: the pixel scale and bow radius are fixed whereas the ratio of shell thickness to bow radius varies.

\subsection{Bow velocity}

Figure 3 shows the effect of changing the bow shock velocity, $v_{\text {bow }}$. Here $b, n_{\mathrm{H}}, \beta$, and $r_{0}$ are kept constant. The bow shock gets brighter when the propagation velocity is increased. In addition a larger part of the wings contributes to the emission. The model with $v_{\text {bow }}=60 \mathrm{~km} \mathrm{~s}^{-1}$ illustrates the result when the velocity increases beyond the critical velocity for which $\mathrm{C}$-shocks can be sustained. When the velocity at the apex is higher than the critical velocity for the given density and B-field, J-shocks are present at the apex. Thus we have a dissociative cap with very little $\mathrm{H}_{2}$ emission. The $\mathrm{C}$-shocks along the wings are bright and due to projection effects $\mathrm{H}_{2}$ emission is found everywhere in the inner region behind the apex. Only the apex region itself shows weak $\mathrm{H}_{2}$ emission.

\subsection{Magnetic field scaling factor}

In Fig. 4 we see the effect of changing the numerical value of the magnetic scaling factor $b$, while keeping the other parameters constant at $n_{\mathrm{H}}=10^{6} \mathrm{~cm}^{-3}$, $v_{\text {bow }}=40 \mathrm{~km} \mathrm{~s}^{-1}, \beta=2.0$, and $r_{0}=71$ pixels $=0.5 R_{\text {bow }}=370$ AU. When $b$ increases, the shock width increases while the $\mathrm{H}_{2}$ brightness decreases. This arises because the magnetic pressure is higher. The shock dissipation and the thermal energy production are therefore spread over a greater distance resulting in a larger extent of the $\mathrm{H}_{2}$ emission zone and a lower temperature and excitation (see e.g. Draine 1980). When $b=1$, the shock at the apex exceeds the maximum allowed speed for a C-shock at the chosen value of $n_{\mathrm{H}}=$ $10^{6} \mathrm{~cm}^{-3}$ and the bow apex has a dissociative cap with little $\mathrm{H}_{2}$ emission as in Fig. 3 for $60 \mathrm{~km} \mathrm{~s}^{-1}$. The $\mathrm{C}$-shocks along the wings are very narrow with shock widths of $24 \mathrm{AU}$. They are just resolved at the chosen pixel scale (5.2 AU). The critical velocity of C-shocks increases when they are embedded in stronger magnetic fields. Therefore, there are no dissociative caps in the models with $b=5$ and $b=8$. The shock width - as defined as the region where the temperature is $>1000 \mathrm{~K}-$ at the apex is $258 \mathrm{AU}$ in the $b=5$ model. At $b=8$ the shock width at the apex increases to $305 \mathrm{AU}$ and the wing emission is weak since the effect of the shock wave is damped substantially by the high magnetic field. At higher magnetic fields, the bow shock is relatively thicker and the emission, while weaker, is relatively more concentrated toward the symmetry axis and apex region. 

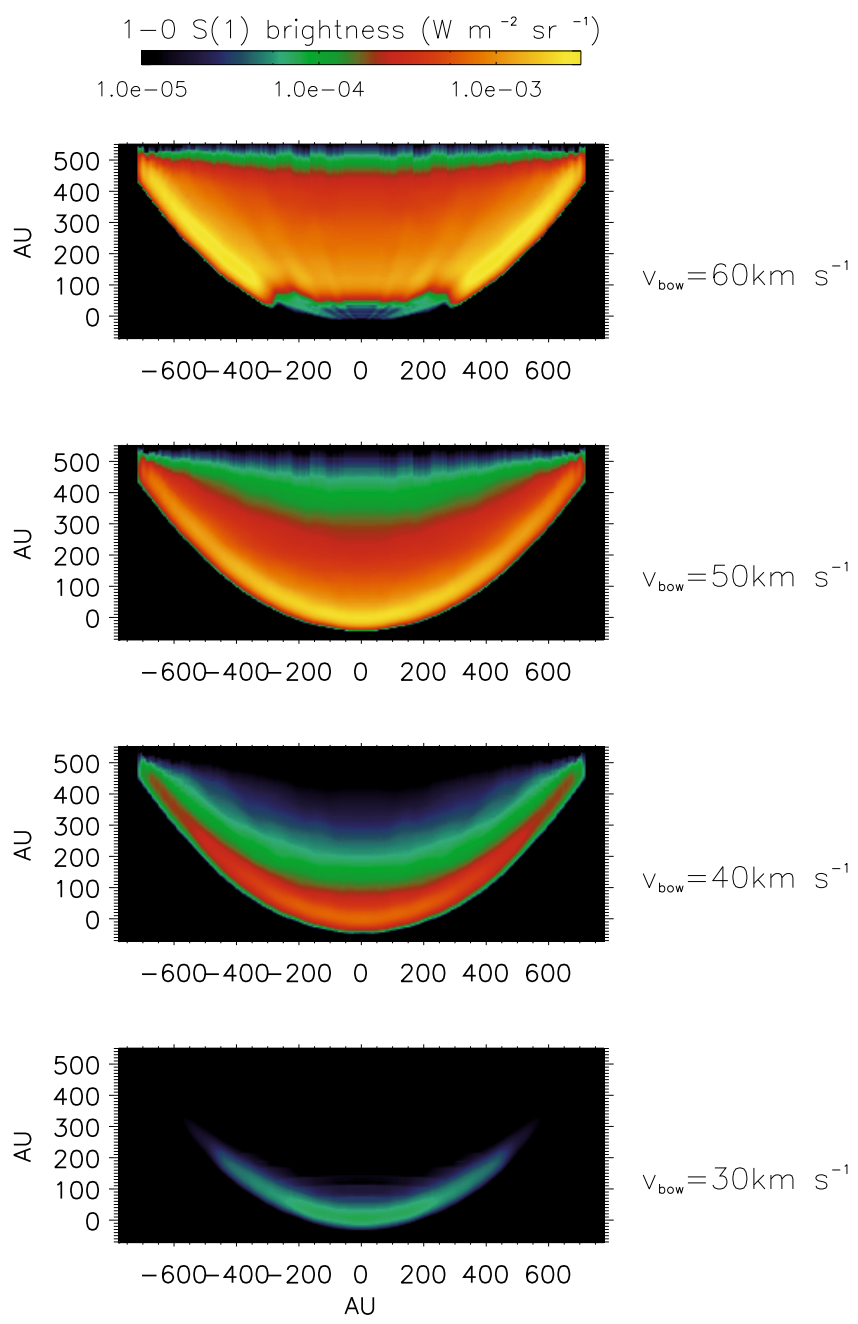

Fig. 3. Brightness, size and morphology as a function of bow velocity. From top to bottom the velocity is $60,50,40$, and $30 \mathrm{~km} \mathrm{~s}^{-1}$. The shock is seen edge-on, $i=90^{\circ}$, with the apex at $(0,0)$. The magnetic field is perpendicular to the flow direction and in the plane of the sky $(\theta=0$, $\phi=0$ ). Other constant input parameters are $n_{\mathrm{H}}=10^{6} \mathrm{~cm}^{-3}, b=5$, $\beta=2.0, r_{0}=200$ pixels $=1040 \mathrm{AU}$. The colour scale is logarithmic. At $60 \mathrm{~km} \mathrm{~s}^{-1}$ the shock velocity at the cap exceeds the critical velocity and the cap becomes dissociative.

The effect of the curvature radius $r_{0}$ may be seen by comparing the model with $b=5$ in the middle panel of Fig. 4, where $r_{0}=0.5 R_{\text {bow }}=370$ AU to the model with $n_{\mathrm{H}}=10^{6} \mathrm{~cm}^{-3}$ in Fig. 2 left column, where $r_{0}=1.4 R_{\text {bow }}=1040$ AU. $r_{0}$ is the only parameter that differs between the two models. When $r_{0}$ is smaller (Fig. 4 middle panel) the bow structure is narrower and the strength of the shocks decreases faster along the wings. In fact, reducing $r_{0}$ is equivalent to shrinking the bow, with all shock parameters remaining the same.

\subsection{Direction of magnetic field with respect to shock propagation}

We now turn to the second group of input parameters and show how the direction of the magnetic field with respect to the flow direction affects the morphology of the $\mathrm{H}_{2}$ emission from a bow shock. The direction of the magnetic field is fixed by two angles: the "obliquity" $\theta$, defined so that $90^{\circ}-\theta$ is the angle of $B$ from the $z$-axis, and the "rotation" angle $\phi$ of the projection of $B$ on the $x-y$ plane (see Fig. 1).

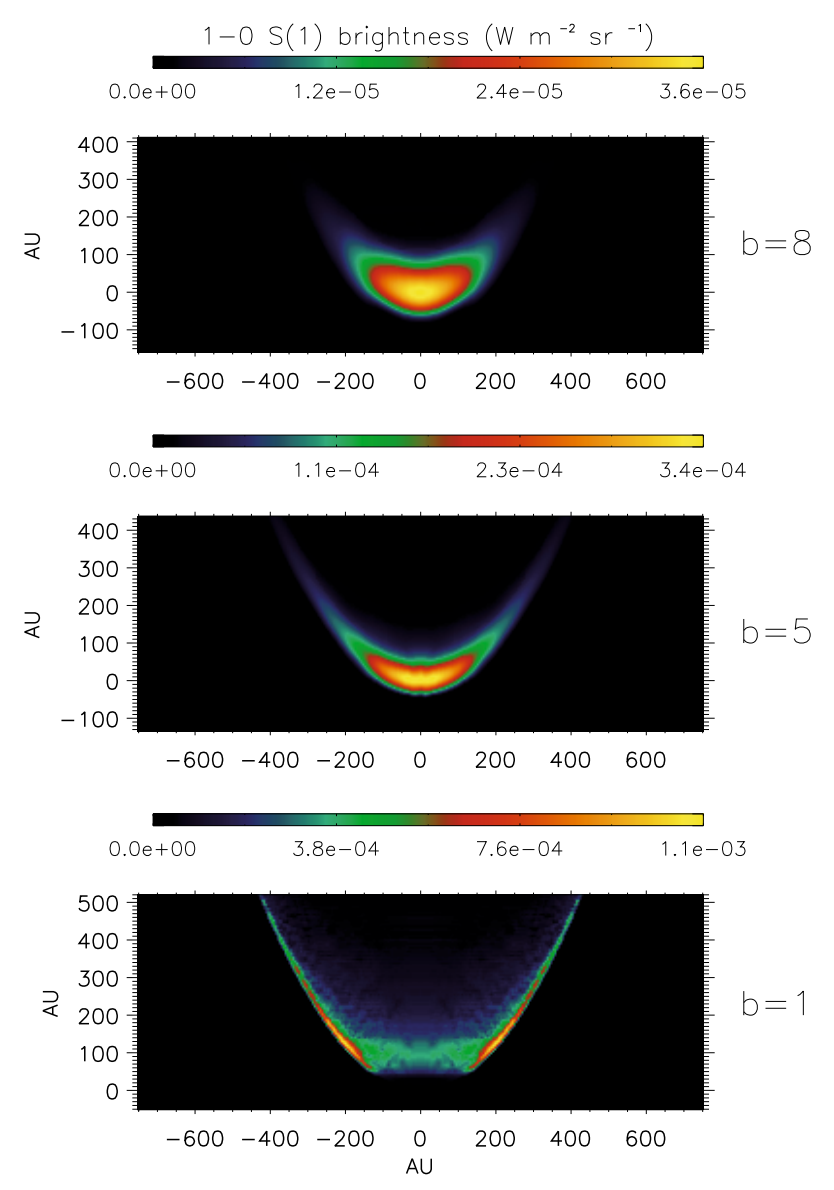

Fig. 4. Brightness and thickness as a function of the magnetic field scaling factor, $b$. From top to bottom, $b=8,5$, and 1 . The shock is seen edge-on, $i=90^{\circ}$, moving along the $y$-axis with the apex at $(0,0)$. The magnetic field is perpendicular to the flow direction and in the plane of the sky $(\theta=0, \phi=0)$. Other parameters remain constant at $n_{\mathrm{H}}=10^{6} \mathrm{~cm}^{3}, v_{\text {bow }}=40 \mathrm{~km} \mathrm{~s}^{-1}, \beta=2.0, r_{0}=71$ pixels $=370 \mathrm{AU}$. The colour scale is linear.

The obliquity angle $\theta$ of the magnetic field with respect to the bow shock axis has a large impact on the 3-dimensional as well as the projected morphology. This is due to the variation of $b_{\|}$ across the bow profile. When the direction of the bow propagation is at an angle to the direction of the B-field, then one side of the bow will experience a $b_{\|}$which is different from that on the opposite side of the bow. On the side which faces into the direction of the B-field, the value of $b_{\|}$will be lower than on the opposite side. This results in brighter, thinner shocks than on the opposite side of the bow, introducing an asymmetry in the brightness distribution.

In Appendix B, the changing morphology is illustrated in detail. There, the standard model $\left(n_{\mathrm{H}}=10^{6} \mathrm{~cm}^{3}, v_{\text {bow }}=40 \mathrm{~km} \mathrm{~s}^{-1}\right.$, $\left.b=5, \beta=2.0, r_{0}=1040 \mathrm{AU}\right)$ is displayed at six angles of inclinations, four different values of $\theta$ and seven values of $\phi$ (see Fig. 1). Here we discuss a limited number of examples and refer to Appendix B for a more extensive view. Note that models with $\phi$ and $180^{\circ}-\phi$ are identical under reflection in the 2 nd axis of the images, except for $\phi=90^{\circ}$ at certain inclinations (see below).

The left column of Fig. 5 illustrates the effect of changing the angle $\theta$ in our standard bow shock model, that is, with $n_{\mathrm{H}}=10^{6} \mathrm{~cm}^{3}, v_{\text {bow }}=40 \mathrm{~km} \mathrm{~s}^{-1}, b=5, \beta=2.0, r_{0}=1040 \mathrm{AU}$. Here the inclination is $i=50^{\circ}$ and $\phi=0^{\circ}$ (ie. magnetic field in the $x-z$ plane). When $\theta=0^{\circ}$ (bottom panel), the B-field is perpendicular to the shock propagation axis and since $\phi=0^{\circ}$ 

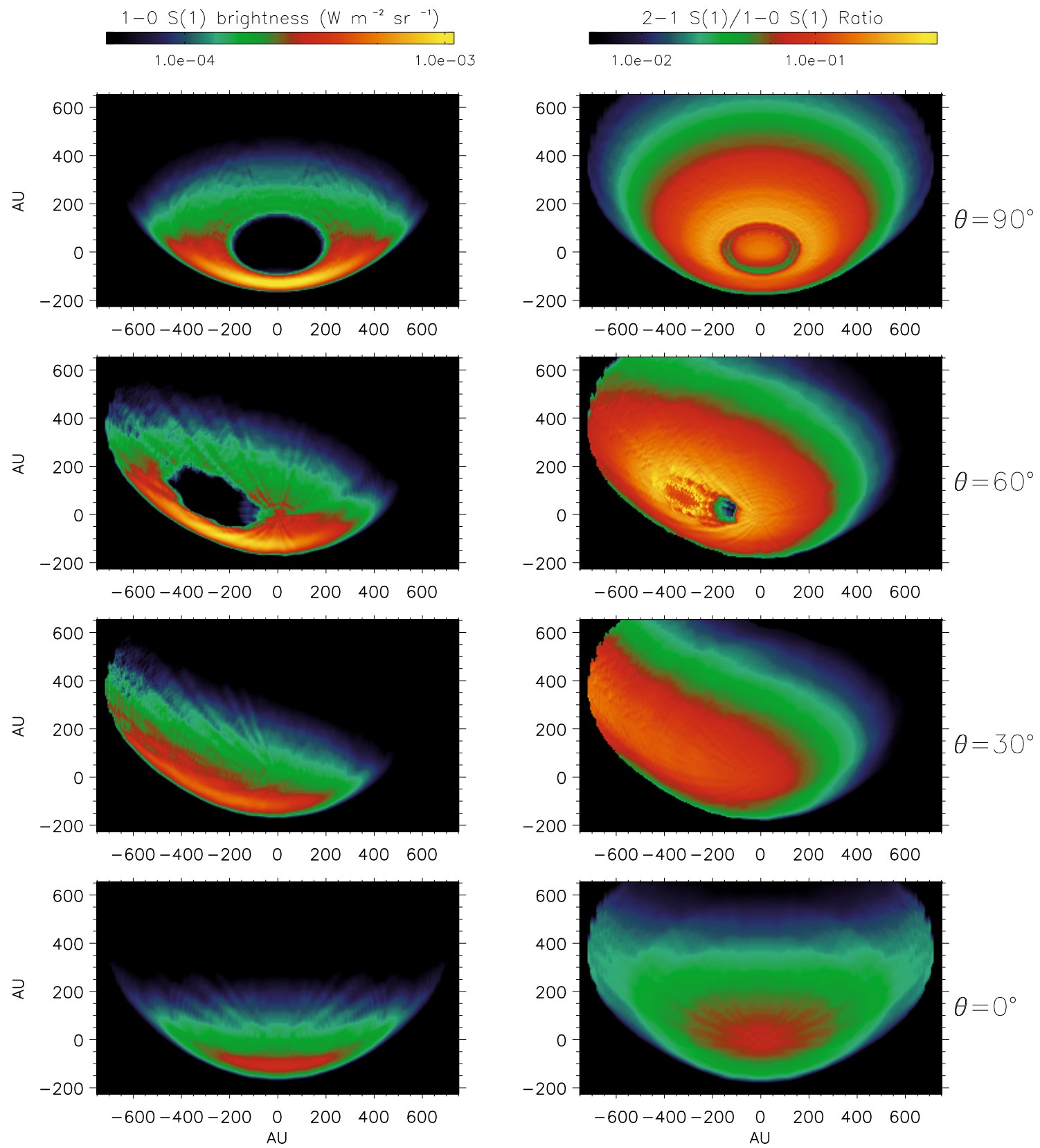

Fig. 5. Left: morphology change as a function of the magnetic field direction $\theta$. From top to bottom, $\theta=90^{\circ}$ (parallel to the direction of shock propagation), $60^{\circ}, 30^{\circ}, 0^{\circ}$ (perpendicular to the direction of shock propagation). The shock is moving at an inclination of $i=50^{\circ}$, the position of the apex is projected onto $(0,0)$. Other parameters remain constant at $n_{\mathrm{H}}=10^{6} \mathrm{~cm}^{3}, v_{\text {bow }}=40 \mathrm{~km} \mathrm{~s}^{-1}, b=5, \beta=2.0, r_{0}=200$ pixels $=1040 \mathrm{AU}$, $\phi=0^{\circ}$. The colour scales are logarithmic. Right: brightness ratio of the $\mathrm{H}_{2} v=2-1 \mathrm{~S}(1)$ line to the $v=1-0 \mathrm{~S}(1)$ line of the models in the left panel.

the value of $b_{\|}$is symmetric with respect to the $y z$-plane. The projected emission from the bow shock is therefore symmetric across $x=0$. When $0^{\circ}<\theta<90^{\circ}$ (exemplified by $\theta=30^{\circ}$ and $\theta=60^{\circ}$, middle panels of Fig. 5), the projected B-field runs from lower-left to upper-right of the image, therefore $b_{\|}$is lower on the negative side of the $x$-axis, where the inclined $\mathrm{B}$-field is more perpendicular to the bow surface, than on the positive side. Thus, the planar shocks and the resulting emission are strongest on the left-hand-side in the figure and the bow shock appears asymmetric. An extreme example may be found for the model with $\theta=60^{\circ}$. A "hole" with little $\mathrm{H}_{2}$ emission is found along a part of the wing (projected into the region around (-300 AU, $100 \mathrm{AU})$ ). This is where the B-field is close to perpendicular to the shock surface and $b_{\|} \sim 0$. The planar shocks are therefore
J-type shocks which are associated here with much weaker $\mathrm{H}_{2}$ emission than $\mathrm{C}$-shocks ${ }^{1}$. When $\theta=90^{\circ}$ (top panel of Fig. 5), the $\mathrm{B}$-field is parallel to the bow axis, and the apex region is inhabited by dissociative J-shocks with weak emission, producing a hole towards $(0,0)$ in the image. The distribution of $b_{\|}$ is now axisymmetric about the bow axis. However, due to the

\footnotetext{
${ }^{1}$ In our standard model the emission from J-shock regions is orders of magnitude weaker than the emission from the surrounding C-shocks. However, the relative strength of the emission in $\mathrm{J}$ - and C-shocks is dependent on the pre-shock density, the b-value, and the bow velocity (Kristensen et al. 2009, in prep.). Thus, bow shocks exist for which the brightness from the J-shocks is comparable to that of the C-shocks. That is, dissociative regions in a bow shock do not always produce a large decrease in $\mathrm{H}_{2}$ emission.
} 
bow inclination of $50^{\circ}$ to the line of sight and the resulting limbbrightening, the projected brightness distribution is not axisymmetric about $(0,0)$ and the $\mathrm{H}_{2}$ emission peak appears projected ahead of the apex.

Figure 5 clearly shows that dissociative J-shocks are not constrained to the apex region of a bow shock. They may also be found along the wings depending on the direction of the magnetic field $(\theta)$ and the geometry of the bow surface $(\beta$ parameter). Another result is that the peak brightness increases with $\theta$. This is because the value of $b_{\|}$decreases at the apex where the highest $v_{\text {shock }}$ is found and the associated planar shocks become stronger. The models in Fig. 5 can be seen at other inclinations in Appendix B.

The effect of changing the "rotation" angle $\phi$ can be seen in Fig. 6 for which $\theta=30^{\circ}$. This corresponds to rotating the bow with respect to the observer, without changing the overall $3 \mathrm{D}$ distribution of $b_{\|}$and shock brightness on the bow surface (fixed by $\theta$ ).

The case $\phi=0^{\circ}$ (3rd panel from top) is the same as discussed in Fig. 5: the emission is strongest to the left from the observer's viewpoint, where $\mathrm{x}$ is negative in Fig. 1. As $\phi$ becomes negative, the B-field rotates in towards the observer, while the strongest planar shocks ( $b_{\|}$lowest) rotate back to the bow side facing away from the observer. They experience more limbbrightening, since the rear part of the bow is more tangential to the line of sight. When $\phi=-90^{\circ}$, the B-field is in the $y z$-plane and points towards the observer, therefore the emission is symmetric with respect to $x=0$ and is strongly limb-brightened. In contrast, when $\phi$ is positive (bottom panel), the brightness decreases since the strongest emission is now on the side of the bow shell facing towards the observer and is thus projected onto a larger area. Note that because of these differences in limbbrightening, maps with $\phi=-90^{\circ}$ and $\phi=90^{\circ}$ are not identical at inclinations different from edge-on (except if $\theta=0^{\circ}$ or $\theta=90^{\circ}$ ), as shown in Appendix B.

\subsection{Inclination}

The projected morphology of a bow shock naturally also depends on the angle at which it moves with respect to the observer. Figure 7 displays our standard shock with parameters $n_{\mathrm{H}}=10^{6} \mathrm{~cm}^{3}, v_{\text {bow }}=40 \mathrm{~km} \mathrm{~s}^{-1}, b=5, \beta=2.0, r_{0}=1040 \mathrm{AU}$, $\theta=30^{\circ}, \phi=30^{\circ}$ at four different inclinations. The apparent aspect ratio of the dimensions of the shock changes significantly when the inclination changes. The emitting region becomes broader as the inclination angle decreases. When the shock is seen edge-on $\left(i=90^{\circ}\right)$ the bow structure is very apparent and the apex is clearly distinguishable by a narrow ridge of strong emission. As the inclination decreases the strong emission ridge gradually disappears. At $i=30^{\circ}$ the strong emission region is more circular and it is distinct from the projected boundary of the bow shock. At $i=0^{\circ}$ the bow shock is seen face-on and the peak emission is located in the centre of the emission region. The brightness increases when the inclination increases because the line of sight traverses a larger number of parcels containing post-shock gas.

From Figs. 5-7, as well as from the numerous examples shown in Appendix B, it should be evident that the direction of the magnetic field and the inclination to the line of sight have a very marked influence on both the morphology of a bow shock and its appearance to the observer. Small changes in the angles of magnetic field with respect to shock propagation can lead to very different shapes and bow shocks with the same intrinsic shape can produce a large number of different observable
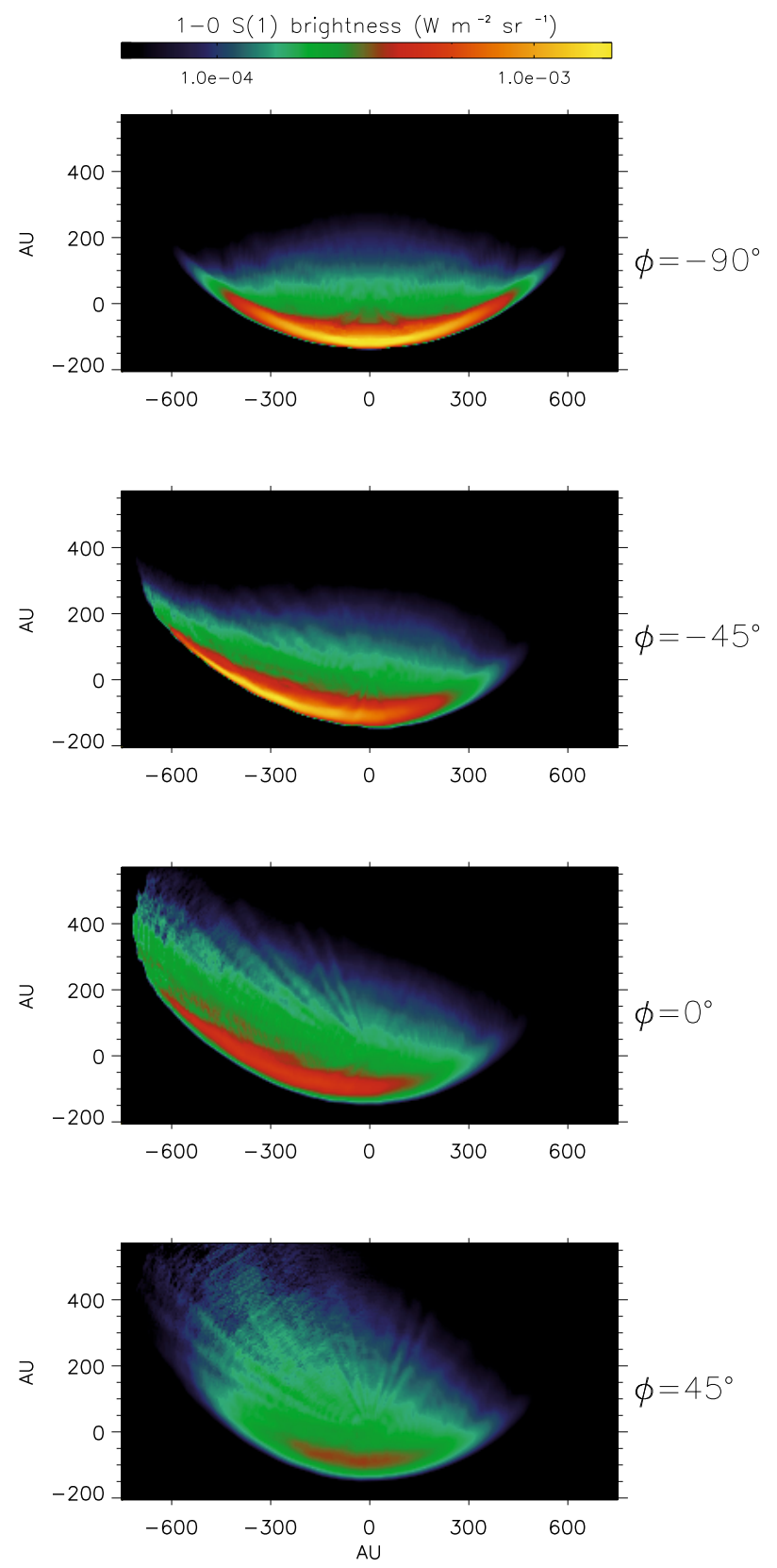

Fig. 6. Morphology change as a function of the magnetic field direction angle $\phi$. From top to bottom, $\phi=-90^{\circ},-45^{\circ}, 0^{\circ}$, and $45^{\circ}$. The shock is moving at an inclination of $i=50^{\circ}$ and the position of the apex is projected onto $(0,0)$. Other parameters remain constant at $n_{\mathrm{H}}=10^{6} \mathrm{~cm}^{3}$, $v_{\text {bow }}=40 \mathrm{~km} \mathrm{~s}^{-1}, b=5, \beta=2.0, r_{0}=1040 \mathrm{AU}, \theta=30^{\circ}$. The colour scale is logarithmic.

emission morphologies. The peak emission may not necessarily be associated with the apex of the bow shock. The peak can be located in front of the projected apex as in Fig. 5 (top left) or displaced along the projected wing as in Fig. 6 (second from top). The combination of $\theta, \phi$ and $i$ determines the exact location. For a full exploration of $\theta, \phi$ and $i$ we again refer to Appendix B.

We therefore reach an important conclusion. A menagerie of shapes of shocked $\mathrm{H}_{2}$ emitting regions greets the observer in Orion and elsewhere. This is nicely exemplified by the very high spatial resolution data for OMC1 presented in Lacombe et al. (2004) obtained with the VLT/NACO adaptive optics system. In images presented there and elsewhere (e.g. Nissen et al. 2007) the presence of rounded structures which are not at all of bow 

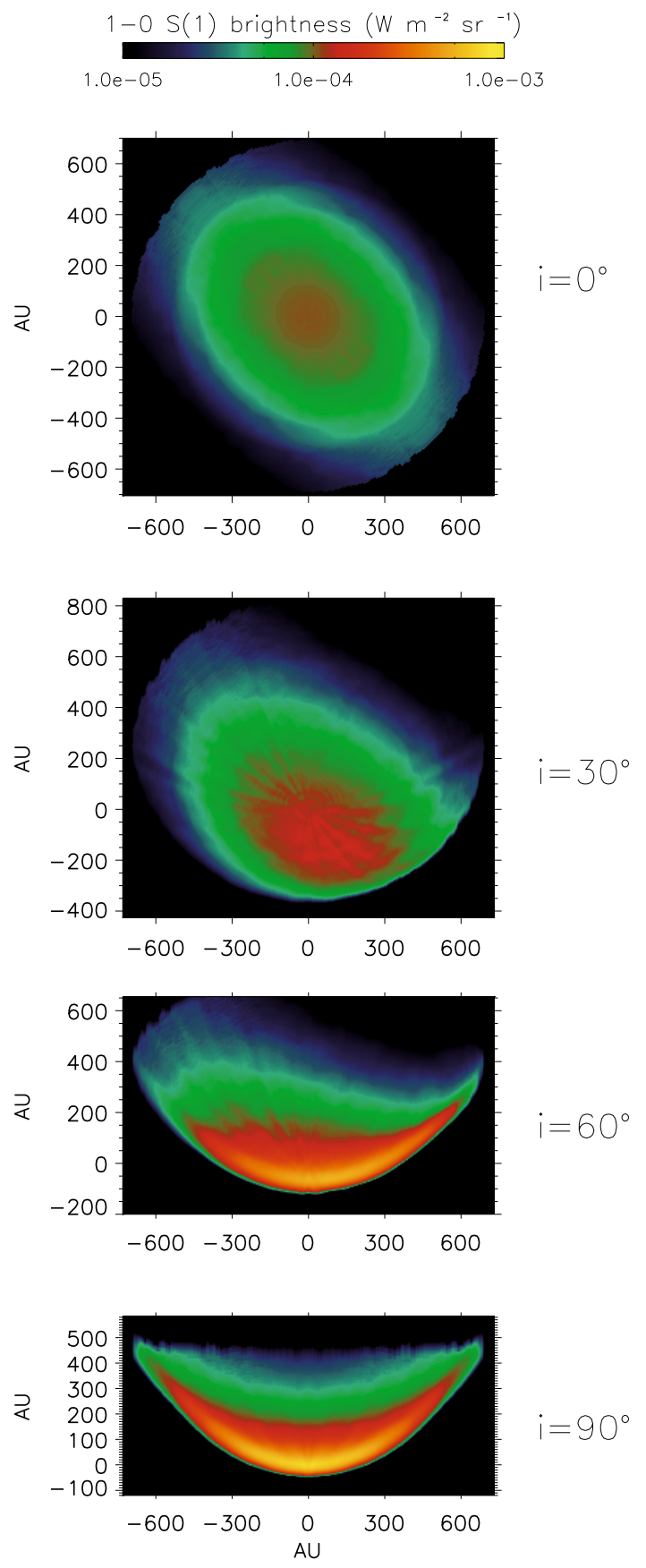

Fig. 7. Morphology change as a function of the inclination angle, $i$. From top to bottom, $i=0^{\circ}$ (face-on), $30^{\circ}, 60^{\circ}, 90^{\circ}$ (edge-on). The position of the apex is projected onto $(0,0)$. Other parameters remain constant at $n_{\mathrm{H}}=10^{6} \mathrm{~cm}^{3}, v_{\text {bow }}=40 \mathrm{~km} \mathrm{~s}^{-1}, b=5, \beta=2.0, r_{0}=1040 \mathrm{AU}$, $\theta=30^{\circ}, \phi=30^{\circ}$. The colour scale is logarithmic.

form, quite symmetric bow-like forms and asymmetric or highly asymmetric but roughly bow-like forms are encountered. This variety of shapes can be attributed very largely to the phenomena which we describe here. Thus broadly speaking we can state that the somewhat confused appearance of shocked zones could be due to shocks moving at a variety of angles to the observer and to the direction the magnetic field. This arises rather naturally in a large cone-angle outflow from a massive star-forming region such as OMC1.

\section{6. $\mathrm{H}_{2}$ emission line ratios}

Observationally, the $\mathrm{H}_{2} v=2-1 \mathrm{~S}(1)$ line is often used together with the $v=1-0 \mathrm{~S}(1)$ line to infer the excitation temperature and hence the shock conditions. The two lines are the brightest $\mathrm{H}_{2}$ lines from the $v=2-1$ and $v=1-0$ vibrational bands, respectively, and hence the easiest to observe.

In the planar shock models the $\mathrm{H}_{2} v=2-1 \mathrm{~S}(1)$ line peaks further downwind than the $v=1-0 \mathrm{~S}(1)$ line and is emitted in a thinner layer because the excitation to the upper level takes place over more restricted physical conditions (Kristensen et al. 2007). This means that the ratio of 2-1 S(1) to 1-0 S(1) emission changes across the bow shell and that projection effects can further alter the relative distribution. Thus a range of values of the line ratio will be present in every model.

In the right column of Fig. 5 we show the line ratio corresponding to the models in the left column. These differ only by the direction of the magnetic field. The first thing to notice is that the line ratio changes significantly between the four models and that a large range of values is associated with any particular model. In the model with $\theta=0^{\circ}$ the line ratio ranges between 0.02 and 0.069 , whereas the line ratio varies between 0.02 and 0.51 when $\theta=60^{\circ}$. At the peak of $v=1-0 \mathrm{~S}(1)$ emission the line ratio is 0.038 at $\theta=0^{\circ}, 0.066$ at $\theta=30^{\circ}$, and 0.13 in the $\theta=60^{\circ}$ and $\theta=90^{\circ}$ models. High line ratio values above 0.2 are usually only seen in J-shock regions as is also evident in Fig. 5 - for example around (-300 AU, $100 \mathrm{AU}$ ) in the $\theta=60^{\circ}$ case - or in Photon Dissociation Regions (PDRs). Here we see that bow shocks can give almost any value depending on the angle of view and magnetic field orientation. The high values are a result of both high values of the line ratio in the planar shock models and projection effects in the 3D model. The J-shocks in the dissociative regions are very narrow (a few pixels in the model) and often much weaker compared to the C-shocks that populate the rest of the bow shock. This makes them very sensitive to line of sight projection. If the line of sight traverses a tail of a C-shock within the bow the line ratio may be altered significantly.

Secondly, we notice that the peak in line ratio is not coincident with the peak of the $v=1-0 \mathrm{~S}(1)$ emission. The line ratio is very sensitive to the shock conditions and decreases with decreasing strength of the $v=1-0 \mathrm{~S}(1)$ emission (Kristensen et al. 2009, in prep.). That is, the $v=2-1 \mathrm{~S}(1)$ line emission decreases faster than the $v=1-0 \mathrm{~S}(1)$ line emission when the shock becomes softer. Thus, the $v=2-1 \mathrm{~S}(1)$ emission is less extended along the wings and it is emitted in a thinner layer than the $v=1-0 \mathrm{~S}(1)$ emission. In short, it is more concentrated towards the location of strong shocks. When the bow shock is inclined to the line of sight the peak of the line ratio will be found close to the projected location of the strong shocks, whereas the peak of $v=1-0 \mathrm{~S}(1)$ emission may be found displaced from this due to limb-brightening. For example, in Fig. 5 lower left corner, the $v=1-0 \mathrm{~S}(1)$ emission peaks at (0 AU, -90 AU) due to limb-brightening, whereas the line ratio peaks at $(0,0)$, which is the projected position of the bow apex. Thus, the relative position of the peak of $v=1-0 \mathrm{~S}(1)$ emission and the $v=2-1 \mathrm{~S}(1) /$ $v=1-0 \mathrm{~S}(1)$ line ratio is very useful for determining the inclination in observations of bow shocks.

\subsection{Excitation temperature}

In this section we investigate the spatial variation of the excitation temperature measured from a number of $\mathrm{H}_{2}$ lines. The excitation temperature is the temperature that reproduces the observed line ratios assuming local thermodynamic equilibrium, 


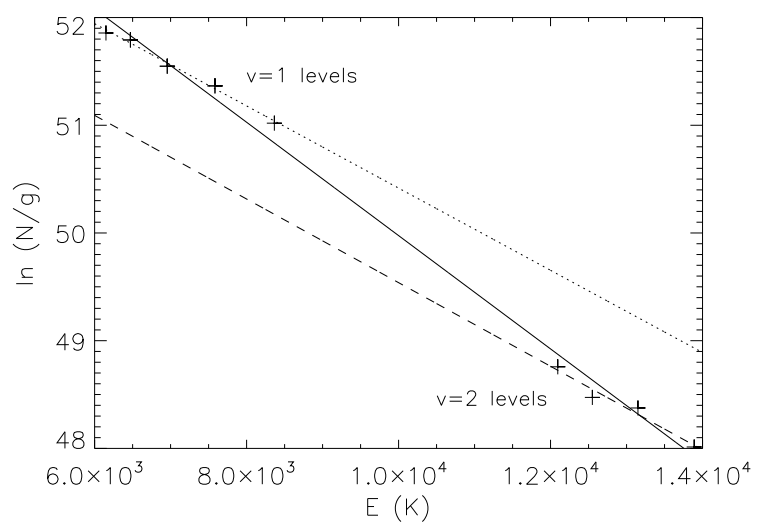

Fig. 8. Example of an excitation diagram from the 3D model in a region where the gas is not completely thermalized. The $v=1-0$ band and the $v=2-1$ band do not line up. Both bands are well fitted individual by the same excitation temperature of $2600 \mathrm{~K}$ (dotted and dashed line, respectively), but with an offset. If both bands are included in the fit (full line), the resulting excitation temperature of $1900 \mathrm{~K}$ is lower than if only one band is used. The input parameters of the model are $n_{\mathrm{H}}=10^{6} \mathrm{~cm}^{-3}$, $v_{\text {bow }}=40 \mathrm{~km} \mathrm{~s}^{-1}, b=5, \beta=2.0, r_{0}=1040 \mathrm{AU}, \theta=30^{\circ}, \phi=0^{\circ}$, and $i=50^{\circ}$.

LTE. Under LTE conditions the column density of the level $(v, J)$ is

$N_{v, J}=\frac{N_{\text {tot }}}{Z(T)} g_{v, J} \exp \left(-E_{v, J} / T\right)$

where $N_{\text {tot }}$ is the total column density, $Z(T)$ is the partition function, $g_{v, J}$ is the degeneracy of the level, $E_{v, J}$ is the level energy expressed in Kelvin and $T$ is the excitation temperature.

Here we calculate the excitation temperature map from maps of the 12 strongest $\mathrm{H}_{2}$ lines in the $K$-band $(v=1-0 \mathrm{~S}(0)-\mathrm{S}(3)$, $\mathrm{Q}(1)-\mathrm{Q}(4)$ and $v=2-1 \mathrm{~S}(0)-\mathrm{S}(3))$. Other lines could have been included as well but the chosen lines constitute the set of lines that can be expected to be detected from integral field spectroscopy observations in the $K$-band (bewaring of atmospheric absorption). At every position in the plane of the sky we find the excitation temperature by fitting to the column densities in Eq. (4). Shock models show that the gas is not in general thermalized. This has the result that lines from the $v=1-0$ band do not line up with lines from the $v=2-1$ band, with the $v=2-1$ band being offset from the $v=1-0$ band. This is illustrated in Fig. 8. Each band by itself is well fitted by a straight line and the temperatures $\left(T_{v}=1-0, T_{v}=2-1\right)$ from the fits are nearly identical. If both the $v=1-0$ and $v=2-1$ band are used in the fit the excitation temperature is $\sim 700 \mathrm{~K}$ lower than $T_{v}=1-0$ or $T_{v}=2-1$. The excitation temperature shows the same trend of decreasing or increasing temperature regardless of which band of the two is used.

The degree of thermalization depends on the gas density, while collisions generate local thermodynamic equilibrium and LTE is therefore more closely achieved in high density gas. Whether the gas reaches LTE also depends strongly on the density of atomic $\mathrm{H}$, as it is more efficient than $\mathrm{H}_{2}$ at collisionally exciting vibrational levels. In our models the initial $\mathrm{H} / \mathrm{H}_{2}$ ratio is fixed at a value determined by the ambient cosmic ray rate. Thermalization would be increased if the pre-shock gas was partly dissociated by previous shocks or by a far-ultraviolet field. We do not consider this possibility here to limit the number of free parameters.

We use the $v=1-0$ band to extract the excitation temperature (Fig. 9) of the models displayed in Fig. 5. In the models in
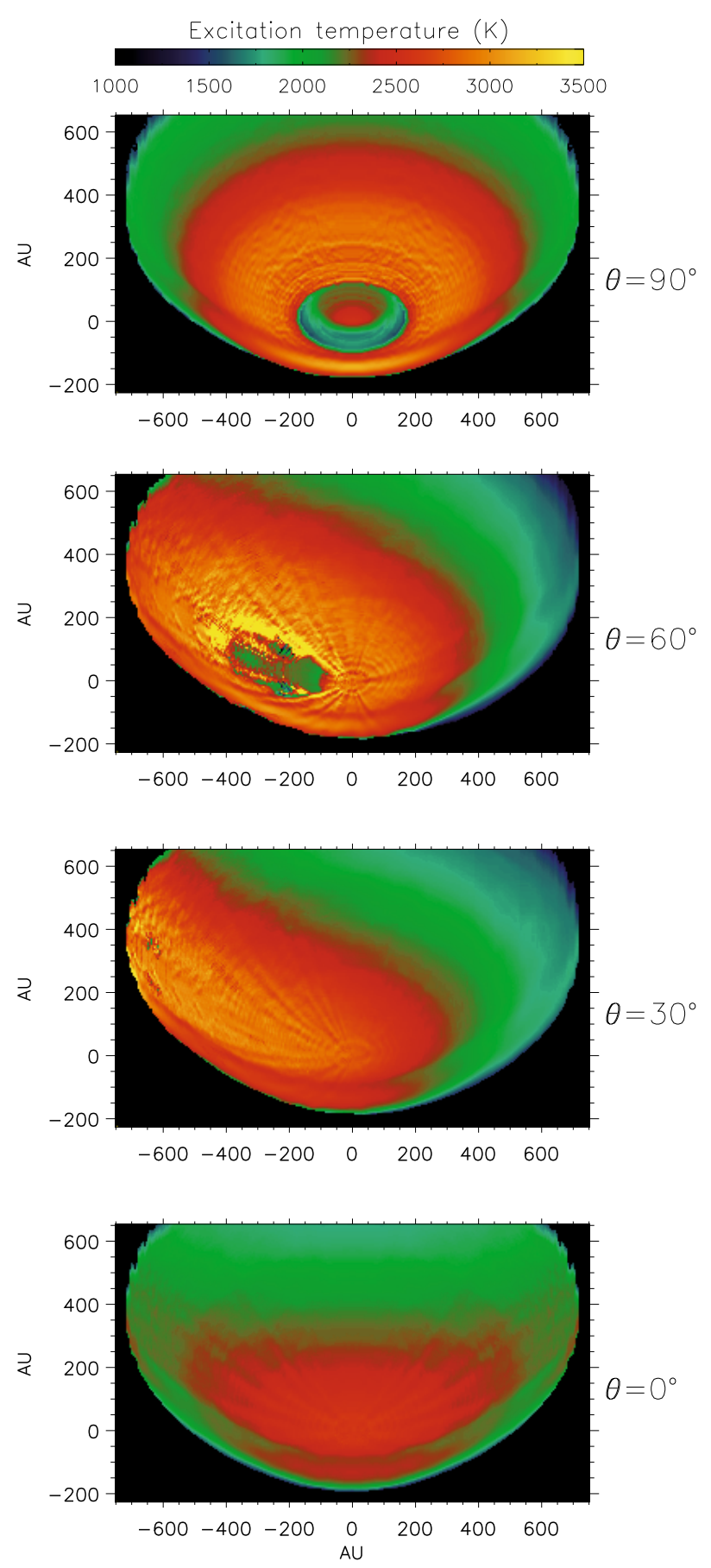

Fig. 9. Excitation temperature as a function of the magnetic field direction $\theta$. Spatial variation of the excitation temperature of $\mathrm{H}_{2}$ derived from ro-vibrational lines in the $v=1-0$ band. The model parameters are the same as in Fig. 5. The colour scale is linear.

Fig. 9 the excitation temperature is $\sim 2300-3300 \mathrm{~K}$ and the temperature is highest in the part of the bow shock where the planar shocks are strongest. This is either at the projected position of the apex or the part of the wings facing toward the magnetic field. The structure of the excitation temperature is the same as that of the $v=2-1 \mathrm{~S}(1) / v=1-0 \mathrm{~S}(1)$ line ratio for the same reasons as discussed for the line ratios. In parts of the dissociative regions, the excitation temperature is very low, which is unexpected since J-shocks are associated with high temperatures. The low temperature stems from the fact that we use only lines from the $v=1-0$ band to calculate the temperature and thus effectively calculate 
M. Gustafsson et al.: 3D model of bow shocks
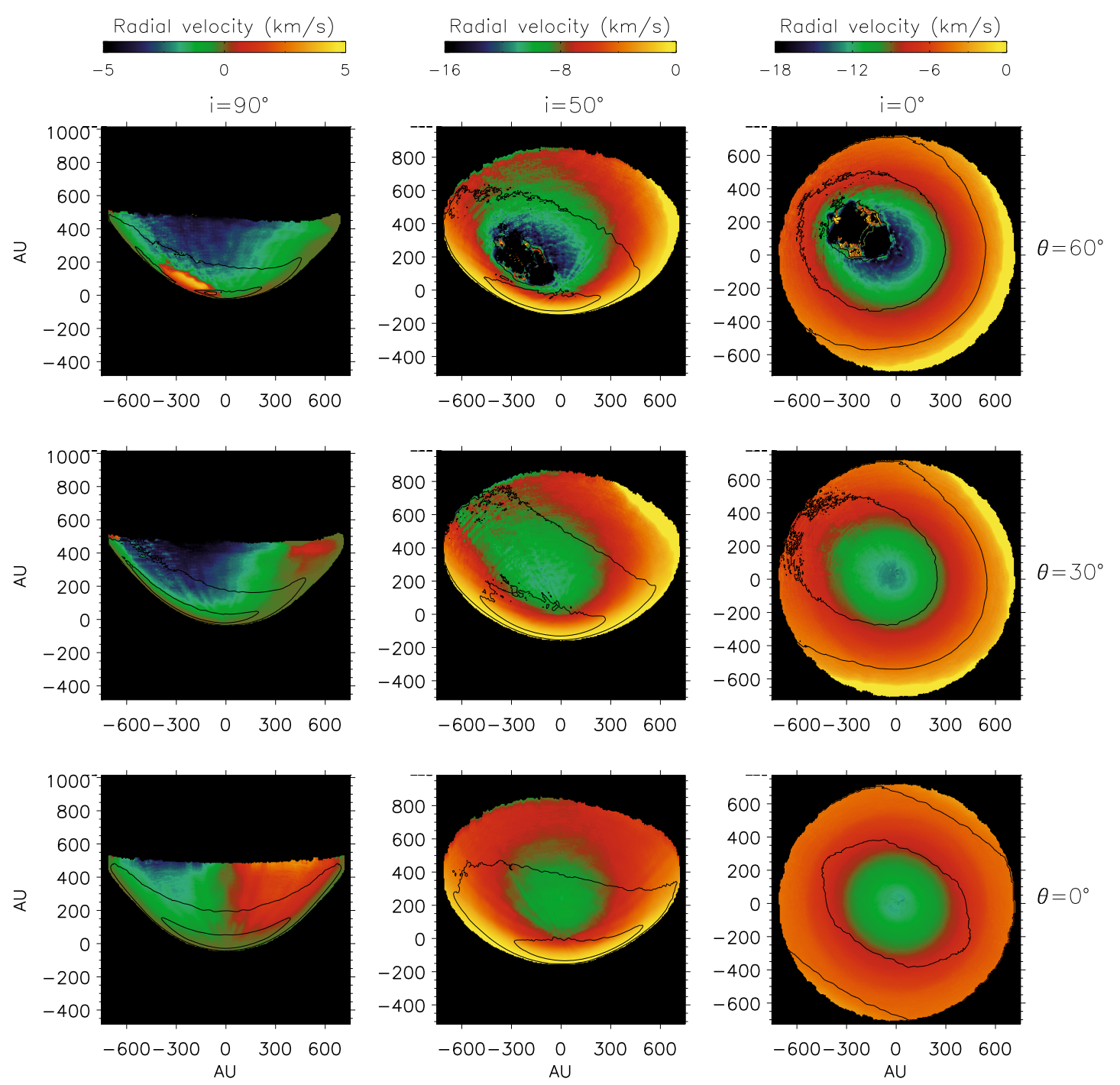

Fig. 10. Centroid radial velocities as a function of inclination and magnetic field direction. From top to bottom $\theta=60^{\circ}, 30^{\circ}, 0^{\circ}$, and from left to right $i=90^{\circ}, 50^{\circ}, 0^{\circ}$. The other parameters are $n_{\mathrm{H}}=10^{6} \mathrm{~cm}^{3}, v_{\text {bow }}=40 \mathrm{~km} \mathrm{~s}^{-1}, b=5, \beta=2.0, r_{0}=1040, \phi=30^{\circ}$. The black contours delineate brightness levels of 0.1 and 0.5 of the maximum brightness of the model. The colour scale is linear.

the rotational temperature of the $v=1-0$ band. In the J-shocks used here the rotational temperature of the $v=1-0$ band is lower than that of the $v=2-1$ band as well as the vibrational temperature, in contrast to $\mathrm{C}$-shocks as discussed above.

\subsection{Radial velocity structure of bow shocks}

The radial velocity structure of bow shocks is of considerable interest for comparison with observational data. With integral field spectroscopy and Fabry-Perot interferometry (Gustafsson et al. 2003; Nissen et al. 2007) it is now possible to obtain 2D maps of the radial velocity of many shocked regions. The velocity information obtained from both integral field spectroscopy and slit-spectroscopy is often also utilised via position-velocity diagrams (e.g., Takami et al. 2006, 2007).

We have calculated the radial velocity as described in Sect. 2 and maps of centroid velocities of the standard model moving at certain angles to the B-field and line of sight are displayed in Fig. 10 for $\phi=30^{\circ}$. The blue-shifted radial velocity is naturally at a maximum when the bow shock is viewed face-on (righthand column in Fig. 10) and the post-shock gas from the apex is moving in the radial direction. The shock velocity at the apex is $40 \mathrm{~km} \mathrm{~s}^{-1}$ in these models, but the bulk of the emitting postshock gas is moving at lower velocities of $\sim 9-15 \mathrm{~km} \mathrm{~s}^{-1}$. The velocity of the post-shock gas is higher when $\theta=60^{\circ}$ than when $\theta=0^{\circ}$. That is because as $\theta$ increases, the magnetic field is closer to the bow axis and the $b_{\|}$component decreases. The shocks are thus narrower and the hot $\mathrm{H}_{2}$ emitting gas reaches velocities closer to the shock speed in the observer's frame. When the shock is moving at an angle $i>0^{\circ}$ to the line of sight the radial velocity decreases and the peak velocity is offset from the peak brightness. The maximum velocity is found behind the maximum brightness within the body of the projected bow shock.

Since we use the centroid radial velocity, where the velocity of each parcel of gas is weighted by the corresponding emission, the velocity structure is very dependent on the location in the line of sight of the emitting gas and may become highly asymmetric. In Fig. $10, \phi=30^{\circ}$. Therefore, when $\theta>0^{\circ}$ the emission is brighter (lower $b_{\|}$) on the left hand quadrant of the front side of the bow. When the bow shock is seen edge-on (left column), the emission is therefore dominated by blue-shifted features. In the model with $\theta=60^{\circ}$ (upper left corner in Fig. 10) the dissociative region is also located in the advancing side of the bow, but the emission there is so weak that the C-shocks in the 
receding wing are found to dominate the emission. This explains the small patch of redshifted velocities around $(-200,100) \mathrm{AU}$ inside the dominantly blueshifted region.

When $\theta=0^{\circ}$ and $\phi=30^{\circ}$, the emission is equally strong in the left hand side of the advancing wing and in the right hand side of the receding wing, and weak on the opposite sides. When such a bow is seen edge-on (lower left corner of Fig. 10), the projected emission map is thus symmetric and the centroid velocities are blueshifted to the left and redshifted to the right of the axis of propagation. This is the same structure as expected from a rotating flow, although the structure clearly does not arise from rotation. Rather, it arises purely from the fact that the emission from the expanding post-shock gas in the bow shock is not cylindrically symmetric around the propagation axis due to the obliquity of the magnetic field from the bow axis. This result shows that any search for rotating outflows needs to be conducted with care and any interpretation must include the effects of the magnetic field on the ambient medium. A velocity change in the outflow perpendicular to the outflow direction may be a projection effect in an expanding outflow lighting up asymmetrically.

\section{Comparison of 1D and 3D models}

The emission structure of a bow shock is determined by geometry, bow velocity, pre-shock density of the ambient material and the magnetic field. When using shock models to reproduce observational data, we are in many circumstances only interested in extracting the bow velocity, and the density of the ambient medium, because that is related to the thrust in outflows from young stars and the driving mechanism of these outflows. In this case we only need to recover the shock conditions at the apex of the bow and it might seem a little excessive to use a 3D model instead of a much simpler 1D model.

In this section, we simulate observations from long slit spectroscopy of the apparent peak of the bow shock, and spatially unresolved observations of the bow shock, in order to test whether these can be used together with 1D models to recover the simulated shock conditions convincingly. That is, we use the simulated brightness maps of a 3D bow shock to extract the $\mathrm{H}_{2}$ brightness from the apparent peak of the model as well as the average projected $\mathrm{H}_{2}$ brightness from the whole bowshock. We compare these to the $\mathrm{H}_{2}$ excitation diagram from the 1D shock at the apex of the 3D model, in which the bow shock conditions are imprinted.

We use our standard 3D model with $\theta=0^{\circ}, \phi=0^{\circ}$, seen at an inclination of $i=50^{\circ}$ (see Fig. 5 lower left panel) as a test case and extract the simulated observable brightness from this. In Fig. 11 we compare the extracted column densities to those of the 1D apex shock of the 3D bow model. First, we notice that the column densities at the brightness peak of the 3D model are higher than the column densities in the 1D apex shock by a factor of $1.5-3$. The position of the peak emission $((0,-80) \mathrm{AU}$ in Fig. 5 lower left panel) is limb brightened as the line of sight traverses a large amount of gas in the bow wing. On the other hand, the averaged column densities from the 3D model are lower than the column densities in the 1D apex shock since they are averages over the full extent of the bow shock where the brightness is greater than $10 \%$ of the peak. Furthermore, the column densities of the apparent apex deviate more from the 1D apex shock, both in terms of absolute values in the $v=1-0$ levels and in the difference between the $v=1$ and the $v=2$ levels, than the average column density of the $3 \mathrm{D}$ model. We thus expect that using the average column densities of the shocked emission to fit the

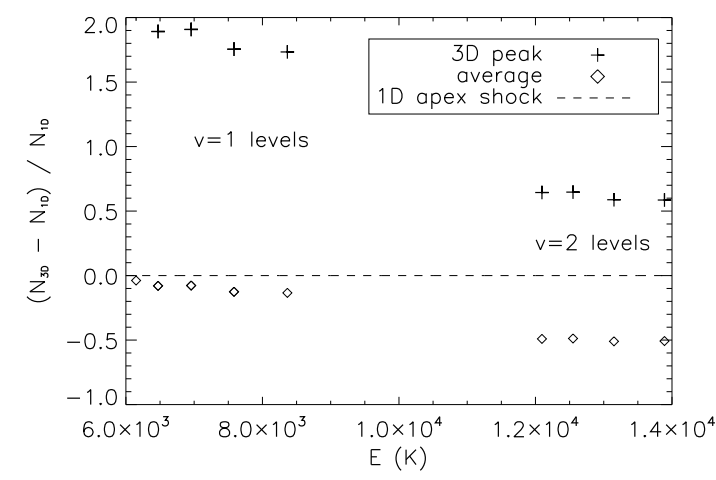

Fig. 11. Deviations in column density of $\mathrm{H}_{2}$ levels of the 3D model with respect to the $1 \mathrm{D}$ shock in the apex. The column densities of the emission peak in the 3D model (+ -signs) are higher than those of the 1D apex shock, whereas the average column density of the 3D model is lower (diamonds). The column densities of the emission peak deviate more from the 1D shock, both in terms of absolute values and in the difference between the $v=1$ and the $v=2$ levels, than the average column density of the $3 \mathrm{D}$ model. The model parameters are $n_{\mathrm{H}}=10^{6} \mathrm{~cm}^{3}$, $v_{\text {bow }}=40 \mathrm{~km} \mathrm{~s}^{-1}, b=5, \beta=2.0, r_{0}=1040 \mathrm{AU}, \theta=0^{\circ}, \phi=0^{\circ}, i=50^{\circ}$.

initial shock conditions gives a better match than using the peak of emission.

Kristensen et al. (2007) presented a grid of 1D shock models, which can be used to find the best fitting model to a set of $\mathrm{H}_{2}$ brightnesses. The average brightness of the inclined bow shock is best matched by a $1 \mathrm{D}$ model with $n_{\mathrm{H}}=10^{6} \mathrm{~cm}^{3}$, $v_{\text {bow }}=45 \mathrm{~km} \mathrm{~s}^{-1}$, and $b=7.5$, whereas a fit to the brightnesses of the apparent apex yields a model with $n_{\mathrm{H}}=5 \times 10^{6} \mathrm{~cm}^{3}$, $v_{\text {bow }}=44 \mathrm{~km} \mathrm{~s}^{-1}$, and $b=10$. This is to be compared to the values of the 3D bow shock model we used (our standard model), $n_{\mathrm{H}}=10^{6} \mathrm{~cm}^{3}, v_{\text {bow }}=40 \mathrm{~km} \mathrm{~s}^{-1}$, and $b=5$. As expected the best match to the shock conditions of the original 3D bow shock model is found by using the average brightness calculated over the whole extent of the projected bow shock. When we use the $\mathrm{H}_{2}$ brightness from the apparent apex both the pre-shock density and the magnetic field scaling factor are significantly overestimated. This result shows that it may be difficult to obtain the true shock conditions with data from longslit spectroscopy that only covers the apex region of a bow shock if the shock is inclined to the line of sight. If observational data is to be compared with $1 \mathrm{D}$ shock models it is best to use the average brightness.

\section{Reproducing observations}

The real value of a 3D bow shock model is the ability to model observations realistically. The Herbig-Haro objects HH7, $\mathrm{HH} 211, \mathrm{HH} 212$, and HH240 have previously undergone detailed 3D modelling (Smith et al. 2003; O'Connell et al. 2004, 2005; Smith et al. 2007) by means of the 3D bow shock model of Smith et al. (2003). In this section we show an example of how our 3D model can be used to model a bow shock observed at subarcsecond resolution in the Orion star forming region.

\subsection{Observational constraints and previous modelling}

The bow shock in question is found in the Orion Molecular Cloud 1, Southwest of the BN object, Source I and the IRc 2 complex. It is part of a blue-shifted outflow system originating in the vicinity of Source I (Nissen et al. 2007, and references therein). It is located at the coordinates 20.'5 W and 6"S of TCC0016 (see Kristensen et al. (2008) for a finding chart) and has previously 
been studied extensively. The great amount of information that is available concerning this object constrains the outputs of the 3D model, which makes the object a good test case.

The magnetic field strength in the OMC1 region has been inferred from several observations. Zeeman measurements show that the magnetic field is $\sim 3 \mathrm{mG}$ near IRc2 (Norris 1984) and that it is of the order of $0.3 \mathrm{mG} \mathrm{24}$ " North of IRc2 (Crutcher et al. 1999). Chrysostomou et al. (1994) estimate the Alfven velocity by measuring the dispersion of polarization angles tracing the magnetic field direction. From this they find that $b \simeq 7$ for $a$ turbulent velocity of $1 \mathrm{~km} \mathrm{~s}^{-1}$.

Kristensen et al. $(2007,2008)$ have previously modelled the shock with 1D and 2D shock models respectively. Kristensen et al. (2007) found that a 1D shock with velocity $\sim 35-40 \mathrm{~km} \mathrm{~s}^{-1}$ and pre-shock density of $n_{\mathrm{H}}=7.5 \times 10^{4} \mathrm{~cm}^{-3}$ could reproduce the brightness of the $v=1-0 \mathrm{~S}(0)$ and $\mathrm{S}(1)$ lines. Using a 2D edge-on model and higher resolution data to reproduce the brightness and emission width along the bow wings, Kristensen et al. (2008) adjusted the shock parameters to a velocity $\sim 50 \mathrm{~km} \mathrm{~s}^{-1}$, pre-shock density of $n_{\mathrm{H}}=5 \times 10^{5} \mathrm{~cm}^{-3}$ and $b=4.8$. This corresponds to a magnetic field strength at the apex of $\sim 3.4 \pm 0.5$ mGauss. From the variations in modelled shock velocity and magnetic field strength along the wings of the bow shock they calculated the position angle of the flow to be $\sim 224^{\circ} \pm 3^{\circ}$ and the position angle of the magnetic field to be $\sim 132^{\circ} \pm 16^{\circ}$. From this, they concluded that the magnetic field is oriented tangentially to the apex. The direction of the magnetic field is consistent with measurements of polarized light that indicate that the magnetic field has a position angle of $\sim 140^{\circ}$ (e.g. Hough et al. 1986; Chrysostomou et al. 1994; Simpson et al. 2006; Tamura et al. 2006). While the 2D model is successful in estimating the direction of the magnetic field it is not capable of simultaneously reproducing the width of the emission and the brightness. The width is underestimated by $\sim 50 \%$ for the best conditions specified in Kristensen et al. (2008).

Using the GriF FP interferometer on CFHT Nissen et al. (2007) measured radial velocities of $\mathrm{H}_{2}$ emitting in the $v=1-0 \mathrm{~S}(1)$ line. They measured a peak radial velocity of $-36 \mathrm{~km} \mathrm{~s}^{-1}$, that is, the object is moving towards us at $36 \pm 1 \mathrm{~km} \mathrm{~s}^{-1}$. Recent proper motion studies performed by Cunningham (2006) indicate that this object has a proper motion of $41 \pm 10 \mathrm{~km} \mathrm{~s}^{-1}$. The full 3D velocity of this object is then $\sim 55 \pm 10 \mathrm{~km} \mathrm{~s}^{-1}$ and the angle with respect to the line of sight is $\sim 50^{\circ} \pm 10^{\circ}$. Therefore, one expects limb-brightening effects that are not taken into account in the 2D edge-on model of Kristensen et al. (2008).

We use here the same data set as Kristensen et al. (2008) for the 3D modelling (Fig. 12). These data were obtained in Dec. 2004 at ESO-VLT with the NACO Fabry-Perot interferometer. Scans were made of the $v=1-0 \mathrm{~S}(1)$ and $\mathrm{S}(0)$ lines as well as the $v=2-1 \mathrm{~S}(1)$ line. The brightness of the three lines was extracted by line-fitting for all spatial positions. The full data reduction has been described in Gustafsson (2006) and Kristensen et al. (2008). The spatial resolution is $\sim 0$.' $^{\prime} 13$ or $60 \mathrm{AU}$ at the distance of OMC1, while the pixel size is $11 \mathrm{AU}$.

\subsection{Fitting procedure}

The 3D model has 8 free parameters (see Table 1) and it is very time consuming to do a large grid-based search of the model that best reproduces the observations if all parameters are included. Therefore, we start by doing some manual exploration in order to limit the parameter space. To facilitate the comparison between observations and model we first rotate the observations so that the flow is moving along the $z$-axis as in the models. Kristensen et al. (2008) estimated the required rotation by fitting a parabola to the ridge of peak emission and found that a rotation of $55^{\circ}$ provided a minimum $\chi^{2}$ value. We adopt this result and rotate the observations clockwise by $55^{\circ}$. Fitting a power-law to the ridge of peak emission yields initial values of the $r_{0}$ and $\beta$ parameters of $400 \mathrm{AU}$ and 1.7, respectively (see Eq. (1)). These estimates of $r_{0}$ and $\beta$ assume that the bow shock is seen edge-on. Therefore they may require later adjustment.

Second, we seek to constrain the inclination angle and the direction of the magnetic field. The morphology of the observed flow helps to constrain the inclination. The emission region is relatively broad with extended emission behind the apex. The apparent apex (the location of peak emission) is, however, clearly distinguishable and the emission falls off more quickly in front of the apex than behind. From the results described in Sect. 3.5 and the figures in Appendix B it is clear that extended emission behind the apex together with a rapid decrease in emission in front limits the inclination angle to $30^{\circ}<i<60^{\circ}$. As an initial value we use the inclination angle indicated by the velocity observations of $i=50^{\circ}$.

Third, we run a mini-grid of 3D models with different values of $\theta$ and $\phi$, which define the orientation of the magnetic field with respect to the shock motion. The goal is to find the direction of the magnetic field that reproduces the morphology of the observed flow, namely the location and the degree of asymmetry of the bright emission. We use the results of Kristensen et al. (2008) as the initial input parameters of the 3D model. We calculate a $3 \mathrm{D}$ model with $n_{\mathrm{H}}=5 \times 10^{5} \mathrm{~cm}^{-3}, v_{\text {bow }}=50, b=5$, $\beta=1.7, r_{0}=400 \mathrm{AU}$ and $i=50^{\circ}$. We explore $\theta=0^{\circ}, 15^{\circ}, 30^{\circ}$, and $45^{\circ}$ and let $\phi$ vary between $90^{\circ}$ and $270^{\circ}$ in steps of $30^{\circ}$. We adopt the same pixel size as in the observation, that is, $11 \mathrm{AU}$.

In order to compare the morphology of the observations and the models we consider only emission stronger than a third of the peak $v=1-0 \mathrm{~S}(1)$ emission, $I_{\max } / 3$. This emission level traces the asymmetry of the emission (see Fig. 5) while being above the noise level in the observational data. The asymmetry of the outer boundary of the $I_{\max } / 3$ emission is determined by $\theta$ and $\phi$. By contrast the inner boundary behind the apex is set by the width of the planar shocks which is given by the combination of $n_{\mathrm{H}}, v_{\text {bow }}$ and $b$. For the moment we seek only to constrain $\theta$ and $\phi$, and therefore we consider only the contour of the outer boundary. We calculate the deviation between the outer boundary of the $I_{\max } / 3$ emission of the observations and the models in the mini-grid. The minimum deviation is found at $\theta=15^{\circ}$ and $\phi=180^{\circ}$.

Fourth, we run a new mini-grid where $\beta, r_{0}, n_{\mathrm{H}}, v_{\text {bow }}$ and $\mathrm{b}$ are varied and $i=50^{\circ}, \theta=15^{\circ}$ and $\phi=180^{\circ}$ are kept constant. The values used in the mini-grid are:

$\beta=1.5,1.7,1.9$

$\alpha \equiv\left(r_{0} / 11 \mathrm{AU}\right)^{1-\beta}=0.02-0.18$, in steps of 0.02,

$n_{\mathrm{H}}=10^{5}, 5 \times 10^{5}, 10^{6} \mathrm{~cm}^{-3}$,

$v_{\text {bow }}=30,40,50,60 \mathrm{~km} \mathrm{~s}^{-1}$

$b=1,2,3,4,5,6$.

We calculate both the $v=1-0 \mathrm{~S}(1)$ and the $v=2-1 \mathrm{~S}(1)$ emission map and fit both to the observations. The $v=1-0 \mathrm{~S}(0)$ emission map was not used in the fit in order to reduce the computation time. This is the last step in the fitting recipe and we now seek to reproduce both the brightness of the $\mathrm{H}_{2}$ lines and the 

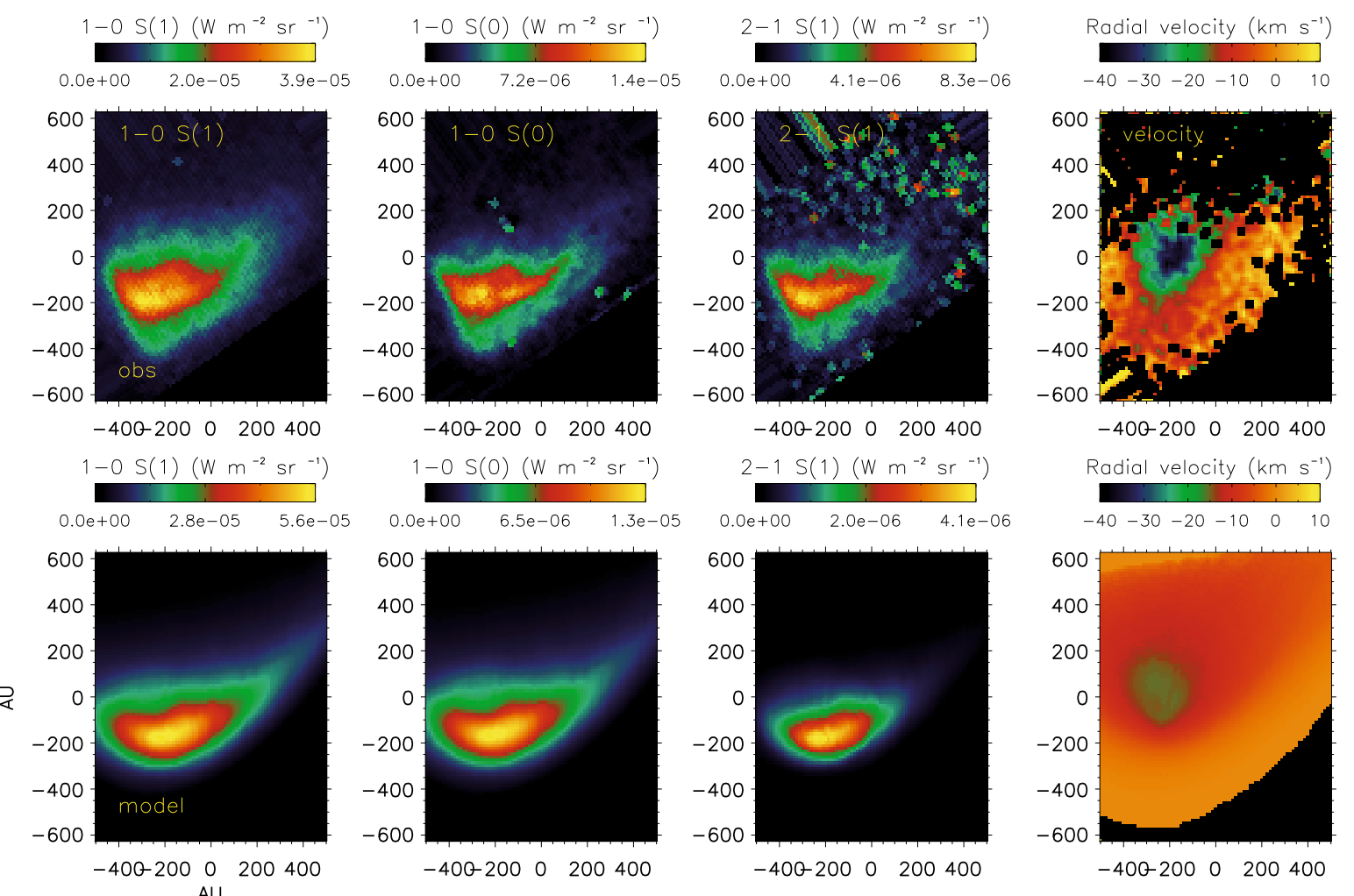

Fig. 12. Brightness of a bow shock in OMC1 observed at ESO-VLT and the corresponding radial velocity map from Nissen et al. (2007) (upper row) compared to the best fit 3D model (lower row). The observational data has been rotated clockwise by $55^{\circ}$. The colour scales are linear.

brightness distribution of the observed flow. To this end we extract five vertical slices from the observations and find the peak brightness and FWHM of the brightness profile in each slice for both the $v=1-0 \mathrm{~S}(1)$ and the $v=2-1 \mathrm{~S}(1)$ lines. Each slice is 5 pixels $=55$ AU wide, they are separated by 3 pixels, and we place one slice on the bow axis, one to the left of the bow axis and three to the right. In the same way we slice up the models and extract peak values and FWHM of the brightness profiles. We then use a $\chi^{2}$ analysis to determine how well the models reproduce the observations. For each model we calculate $\chi^{2}=\sum\left(\frac{X_{\text {obs }}-X_{\text {model }}}{\sigma_{\text {obs }}}\right)^{2}$ where $X_{\text {model }}$ and $X_{\text {obs }}$ refer to the modelled and observed properties, respectively, and $\sigma_{\mathrm{obs}}$ is the observed uncertainty. The parameters of the best fit model, that is, the model that minimises $\chi^{2}$, are summarised in Table 1 . As a final check we run models with the best fit parameters of $\beta, r_{0}, n_{\mathrm{H}}$, $v_{\text {bow }}$ and $\mathrm{b}$ and different values of $i, \theta$ and $\phi$. We do this to ensure that the fitted combination of $\beta, r_{0}, n_{\mathrm{H}}, v_{\text {bow }}$ and $\mathrm{b}$ does not cause the morphology to be better fitted by a different inclination angle and magnetic field direction. This is not the case.

\subsection{Modelled versus observed maps}

The brightness maps of the $v=1-0 \mathrm{~S}(1)$, the $v=1-0 \mathrm{~S}(0)$ and the $v=2-1 \mathrm{~S}(1)$ lines from the observations, as well as the associated radial velocity map from Nissen et al. (2007) are compared to the best fit model in Figs. 12 and 13. The brightness of the model is in good agreement with the observations. The modelled brightness of the $v=1-0 \mathrm{~S}(1)$ line is about $30 \%$ too high compared to the observations, whereas the $v=2-1 \mathrm{~S}(1)$ brightness is underestimated by a factor of two. Note that the fit to the $v=2-1 \mathrm{~S}(1)$ brightness could be improved by increasing the $\mathrm{H} / \mathrm{H}_{2}$ ratio. This would create more collisions and thereby LTE conditions would be more closely achieved. However, we
Table 1. Model parameters of the model that best reproduces the observations.

\begin{tabular}{cc}
\hline \hline Parameter & Best fit model \\
\hline$n_{\mathrm{H}}\left(\mathrm{cm}^{-3}\right)$ & $10^{5}$ \\
$v_{\text {bow }}\left(\mathrm{km} \mathrm{s}^{-1}\right)$ & 50 \\
$b$ & 3 \\
$\beta$ & 1.7 \\
$r_{0}(\mathrm{AU})$ & 610 \\
$\theta\left(^{\circ}\right)$ & 15 \\
$\phi\left({ }^{\circ}\right)$ & 180 \\
$i\left(^{\circ}\right)$ & 50 \\
\hline
\end{tabular}

Notes. $\mathrm{H} / \mathrm{H}_{2}=4.1 \times 10^{-5}$.

fix the $\mathrm{H} / \mathrm{H}_{2}$ ratio by the cosmic ray rate. This is in contrast to Smith et al. (2003) who use the $\mathrm{H} / \mathrm{H}_{2}$ ratio as a free parameter. The $v=1-0 \mathrm{~S}(0)$ brightness, which was not included in the fit, is only $\sim 8 \%$ lower than the observation.

The width of the shock as well as the general morphology of the flow is also very well reproduced for all three emission lines. From Fig. 13 it is evident that the observed and modelled contours of $20 \%, 50 \%$ and $80 \%$ of the maximum brightness agree to a great extent. The asymmetric tail of emission extending towards the upper right corner is also well reproduced by the model. The ability of the 3D model to reproduce simultaneously the brightness and the width of the emission is a substantial improvement over existing 1D and 2D shock models.

The radial velocity in the model peaks at the same position as the observed velocities, that is, behind the apex. This is very encouraging and confirms that at least the inclination of the model is correct. The modelled velocities are, however, lower than the observed by $\sim 20 \mathrm{~km} \mathrm{~s}^{-1}$ towards the apex. A possible 

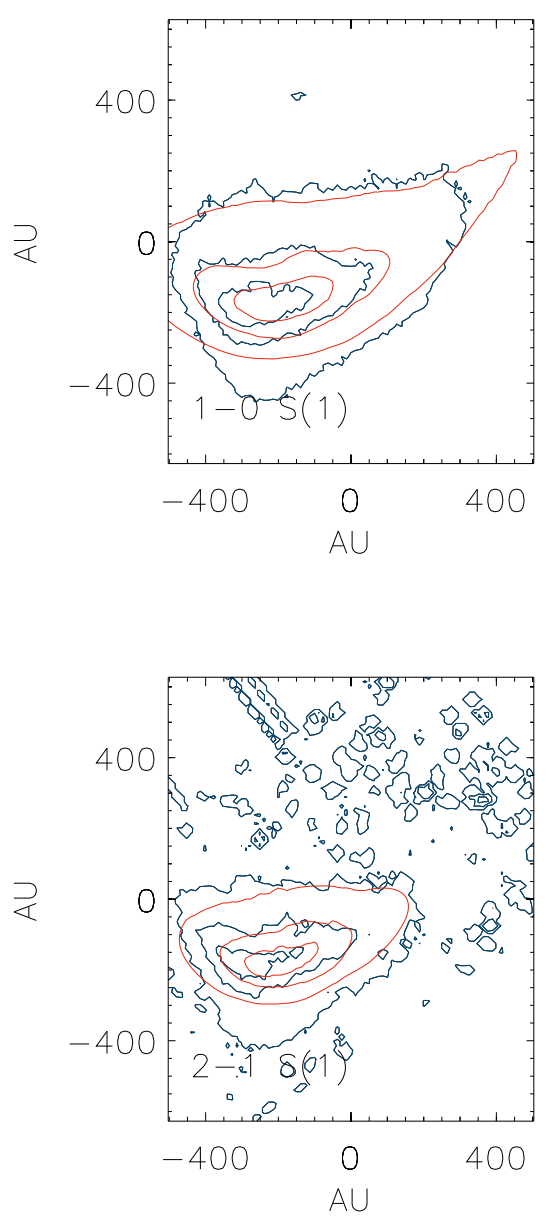
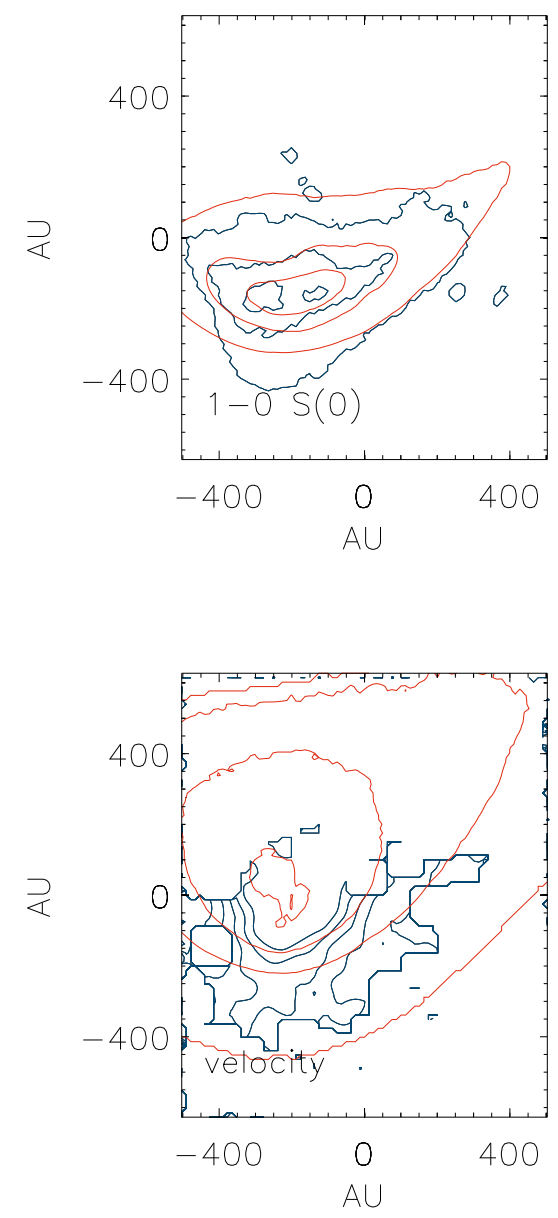

Fig. 13. Contours of brightness and radial velocity from the observations (blue) and the model (red). The contour levels in the three brightness plots are $0.2,0.5$, and 0.8 times the maximum brigthness. In the plot of radial velocity the contours delineate $-20,-15,-10$, -5 , and $0 \mathrm{~km} \mathrm{~s}^{-1}$. explanation may be that the apex region contains an additional contribution from a Mach disk, as mentioned in Kristensen et al. (2008).

We find that the magnetic field is oriented at a small angle $\theta=15^{\circ}$ from the tangential plane of the apex. This is consistent with the results in Kristensen et al. (2008), who found that the magnetic field is tangential to the bow apex. With $\phi=180^{\circ}$ and $i=50^{\circ}$, we predict that the B-field projected in the plane of the sky makes an angle of $78^{\circ}$ from the bow axis, ie. a PA angle of $55+78=133^{\circ}$ from North after correcting for the rotation applied to the bow image. This is in excellent agreement with the results in Kristensen et al. (2008) and with polarization measurements that indicate a PA $\sim 140^{\circ}$ in this region (e.g. Hough et al. 1986; Chrysostomou et al. 1994; Simpson et al. 2006; Tamura et al. 2006). The $b=3$ value of our best fit model is lower than the $b=4.8$ estimated by Kristensen et al. (2008) and the scaling factor $b \simeq 7$ inferred by Chrysostomou et al. (1994), but we note that the latter was estimated in a slightly different region (southeast of Irc2) and could be uncertain by a factor 2 . For our preshock density of $10^{5} \mathrm{~cm}^{-3}, b=3$ corresponds to a magnetic field strength of $\sim 1 \mathrm{mG}$. We then predict a line of sight component of $\pm 0.16 \mathrm{mG}$ for $\phi=180^{\circ}$ and $i=50^{\circ}$. In comparison, Zeeman detections range from $0.3 \mathrm{mG}$ to $3 \mathrm{mG}$ around Irc2 (Crutcher et al. 1999; Norris 1984). Since bowshocks bright in $\mathrm{H}_{2}$ will tend to be found preferentially in areas of lower magnetic field strength, the agreement is again quite good.

Thus, with the 3D model we can simultaneously reproduce the brightness levels in three $\mathrm{H}_{2}$ lines as well as the shape and size of the emitting region. The radial velocity map of the model is in qualitative agreement with the observed. Furthermore, the predicted magnetic field direction is in excellent agreement with the observed one while the scaling factor $b$, and amplitude of the magnetic field appear consistent with the range of observed values.

\section{Concluding remarks}

We have presented a three-dimensional model of interstellar bow shocks propagating in a homogeneous molecular medium with a uniform magnetic field. The model is constructed by associating a one-dimensional planar shock with every point on a threedimensional bow skeleton. Improvements over previous work are the inclusion of a highly sophisticated chemical reaction network, which is essential for predicting realistic emission brightness, and a (simplified) treatment of motion parallel to the bow surface.

With the 3D model we can predict the projected morphology in the plane of the sky of a bow shock moving at an arbitrary inclination and with a magnetic field inclined at an arbitrary angle. In this paper we have focussed on rovibrational molecular hydrogen emission, but emission maps of atomic species and other molecular species can also be predicted. To limit the number of free parameters, we assume here that the preshock ionization and the atomic $\mathrm{H}$ fraction are set by the cosmic ray flux, i.e. the preshock gas is assumed well screened from any far-ultraviolet source.

The shock width depends primarily on density, with increasingly narrower shocks for higher density. The appearance in the plane of the sky of a bow shock changes dramatically if the ratio of the shock width to the curvature of the bow shell changes. If the shock width is large compared to the local radius of 
curvature, the emission region is very compact and the bow structure is not discernible.

The magnetic scale factor, $b=B(\mu \mathrm{G}) / \sqrt{n_{\mathrm{H}} / \mathrm{cm}^{-3}}$, also strongly influences the shock width. When $b$ increases, the shocks get softer and more extended. When the propagation velocity, $v_{\text {bow }}$, is increased the shocks become stronger and a larger part of the wings lights up. If $v_{\text {bow }}$ is increased above the critical velocity at which a $\mathrm{C}$-shock can be sustained for the given density and b-value, a dissociative cap inhabited by dissociative J-shocks develops.

The morphology of the emission from a bow shock is highly dependent on the orientation of the magnetic field and the inclination of the flow. The planar shocks are strongest on the part of the bow surface that faces towards the magnetic field, where the parallel field strength $b_{\|}$is lowest. The result is that the morphology can be highly asymmetric. Together with inclination effects this means that bow shocks can appear in many different guises and they do not necessarily - or even generally - show a characteristic bow shape. Thus broadly speaking we can state that the somewhat confused appearance of shocked zones in star forming regions could be due to shocks moving at a variety of angles to the observer and to the direction of the magnetic field.

J-shocks may also be found in the part of the wing of the bow where $b_{\|} \sim 0$ leading to a shock velocity in excess of the critical velocity. The molecular hydrogen emission from dissociative shocks is often much weaker than that from C-shocks. Thus, dissociative regions in the bow may show up as depressions in the brightness. Depressions due to dissociative caps at the apex, mentioned above, can appear behind a leading ridge of emission if the bow shock is not moving in the plane of the sky.

The $\mathrm{H}_{2} v=2-1 \mathrm{~S}(1)$ line is emitted efficiently under more restricted physical conditions and therefore in a thinner layer than the $v=1-0 \mathrm{~S}(1)$ line. The ratio of the two lines is therefore variable across the flow, and the spatial offset between the peaks of the lines may be used to estimate the inclination of the flow.

We have also computed centroid radial velocity maps of bow shocks. The radial velocity is naturally maximum when the shock is seen face-on and decreases when the inclination increases. The radial velocity peaks behind the brightest region of the bow shock when the flow is seen at an inclination different from face-on. Under certain circumstances the radial velocity of an expanding bow shock can show the same signatures as a rotating flow. In this case a velocity gradient perpendicular to the outflow direction is a projection effect of an expanding bow shock lighting up asymmetrically due to the orientation of the magnetic field.

Using the 3D model we have tested whether it is possible to infer the underlying shock conditions at the bow apex by fitting 1D models to line brightnesses extracted from the projected maps. Our results indicate that the apex shock conditions are better retrieved if the average line emission over the bow is used in the $1 \mathrm{D}$ shock fit, rather than the line emission at the brightness peak. The former can be obtained by spatially unresolved observations, while longslit spectroscopy can supply the latter.

Finally, we have demonstrated the ability of the 3D model to reproduce observational data on a small-scale bow shock in Orion. With the 3D model we can reproduce the brightness levels in three $\mathrm{H}_{2}$ rovibrational lines, as well as the asymmetric shape and the thickness of the emitting region. Previous modelling involving $1 \mathrm{D}$ and $2 \mathrm{D}$ models has not been able to reproduce both the brightness and the shock width - and naturally not the appearance of the shock to the observer. The radial velocity map of the 3D model is in qualitative agreement with observations and the predicted direction and strength of the magnetic field

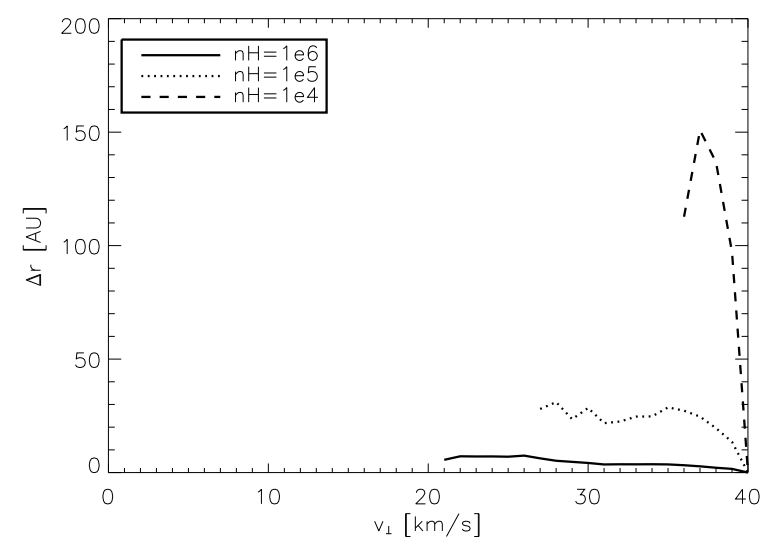

Fig. A.1. The radial displacement (see Eq. (A.7)) of the peak $v=$ 1-0 $\mathrm{S}(1) \mathrm{H}_{2}$ emissivity from the $z$-axis, for planar shocks along a bow with $b_{\|}=5, v_{\text {bow }}=40 \mathrm{~km} \mathrm{~s}^{-1}$, and various preshock densities. Only models with $T_{\max }>1000 \mathrm{~K}$ are displayed.

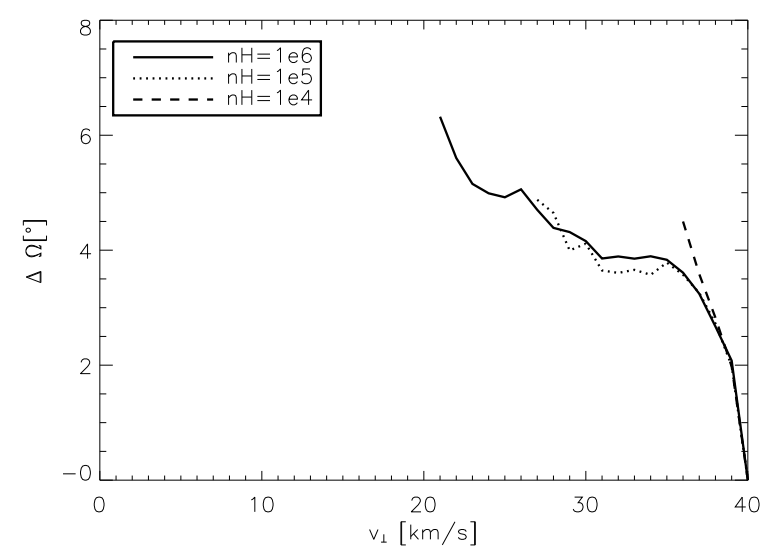

Fig. A.2. The angular displacement in degrees (see Eq. (A.8)) of the peak $v=1-0 \mathrm{~S}(1) \mathrm{H}_{2}$ emissivity from the $z$-direction, for the same models as in Fig. A.1.

fit into the pattern suggested by polarization maps and Zeeman splitting.

The 3D model thus enables us to determine the shock conditions with an accuracy that was not previously possible with $1 \mathrm{D}$ or $2 \mathrm{D}$ shock models. Furthermore, it allows us to estimate the viewing angle and the direction of the magnetic field with respect to the shock propagation in addition to density, shock velocity, and magnetic flux density. These capabilities are crucial for studying the physics and geometry of outflows in star forming regions and for understanding the role that magnetic fields play in shaping the observed $\mathrm{H}_{2}$ features.

Acknowledgements. We would like to thank the referee, Alex Raga, for very useful comments and suggestions considering the inclusion of tangential cooling in the model. M.G. would like to acknowledge the support of Aarhus University during a longer visit.

\section{Appendix A: Validity of our approximations}

In the 3D model, we include the displacement of the postshock gas parallel to the bow surface in an approximate but internally self-consistent way, through several assumptions that we discuss below. 
M. Gustafsson et al.: 3D model of bow shocks

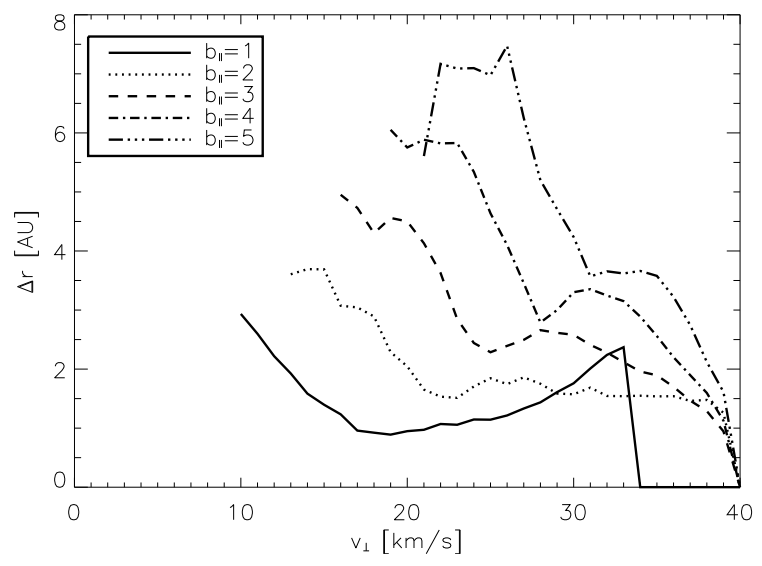

Fig. A.3. The radial displacement (see Eq. (A.7)) of the peak $v=$ 1-0 S(1) $\mathrm{H}_{2}$ emissivity from the $z$-axis, for planar shocks along a bow with $n_{\mathrm{H}}=10^{6} \mathrm{~cm}^{-3}, v_{\text {bow }}=40 \mathrm{~km} \mathrm{~s}^{-1}$, and various values of $b_{\|}$. Only models with $T_{\max }>1000 \mathrm{~K}$ are displayed.

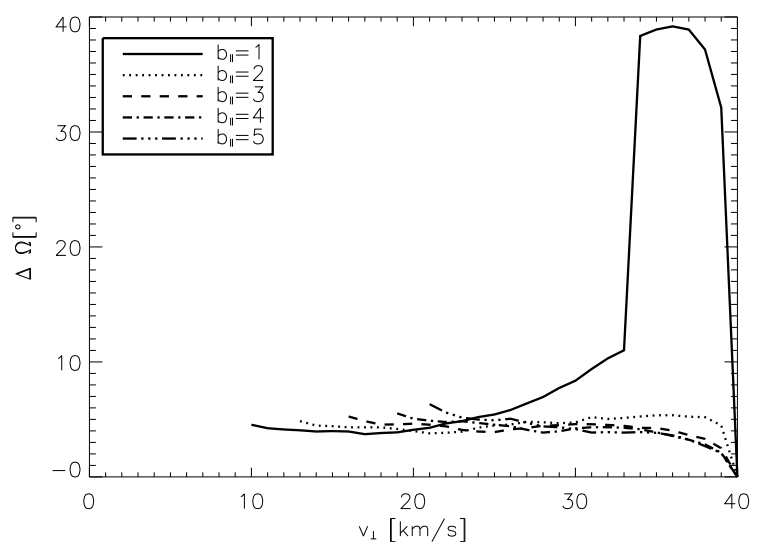

Fig. A.4. The angular displacement in degrees (see Eq. (A.8)) of the peak $v=1-0 \mathrm{~S}(1) \mathrm{H}_{2}$ emissivity from the $z$-direction, for the same models as in Fig. A.3.

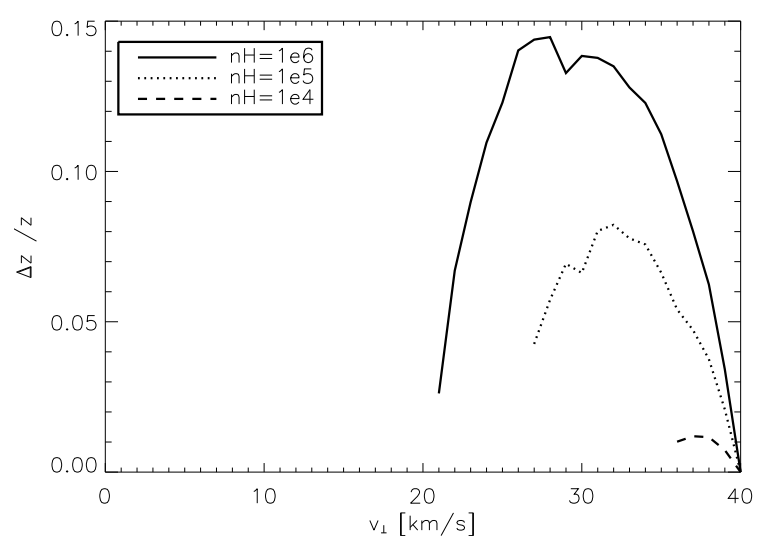

Fig. A.5. The relative error in the $z$-position (see Eq. (A.11)) of the peak $v=1-0 \mathrm{~S}(1) \mathrm{H}_{2}$ emissivity for the same models as in Fig. A.1.

\section{A.1. z-axis approximation}

First, we use the fact that $\mathrm{H}_{2}$ rovibrational emission in C-shocks occurs before the gas has slowed down significantly and assume, for constructing our emission maps, that the post-shock gas of the individual planar shocks flows along the z-axis in the cooling region. We keep, however, the exact value of its velocity vector to compute maps of centroid radial velocities. Since the streamlines are approximated as cylinders, our neglect of adiabatic

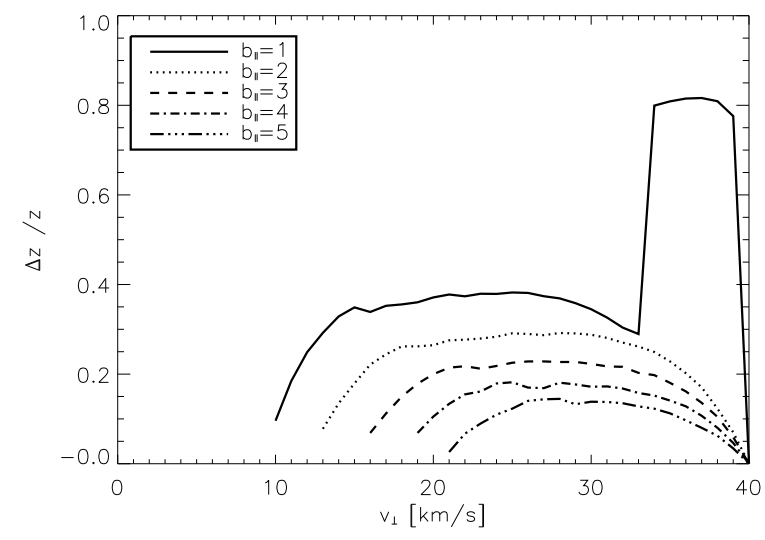

Fig. A.6. The relative error in the z-position (see Eq. (A.11)) of the peak $v=1-0 \mathrm{~S}(1) \mathrm{H}_{2}$ emissivity for the same models as in Fig. A.3.

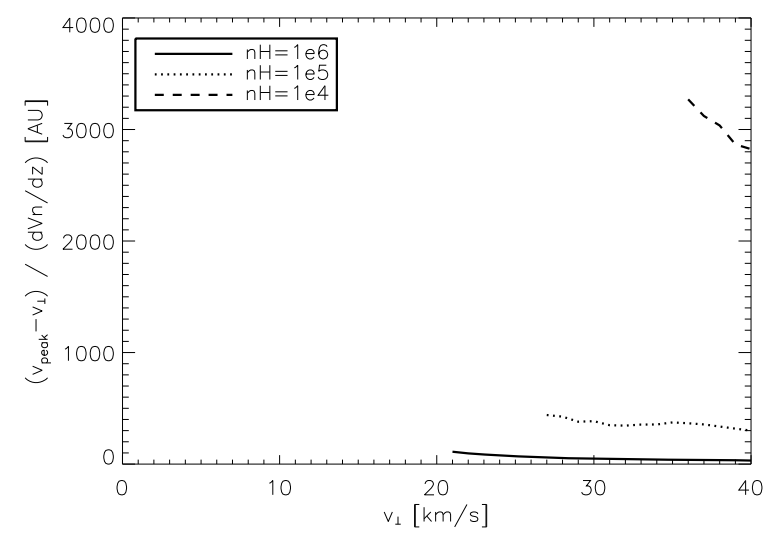

Fig. A.7. Characteristic 1D velocity variation scale for planar models with $b_{\|}=5, v_{\text {bow }}=40 \mathrm{~km} \mathrm{~s}^{-1}$, and various pre-shock densities. Only models with $T_{\max }>1000 \mathrm{~K}$ are displayed.

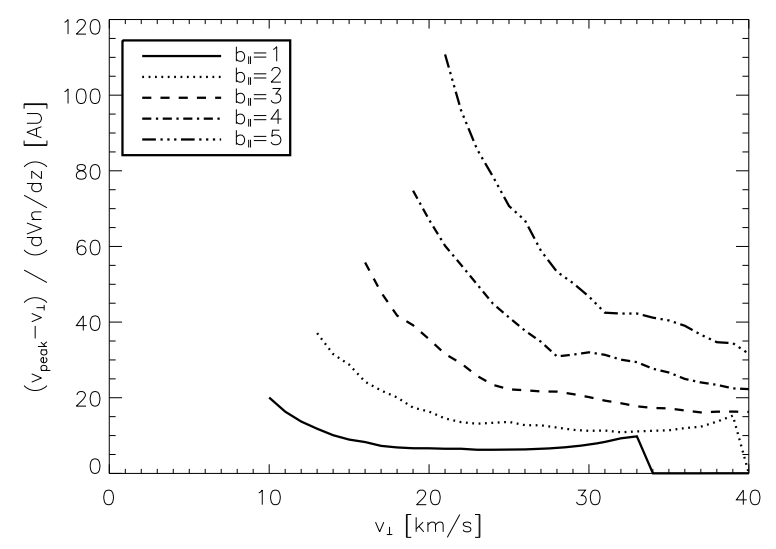

Fig. A.8. Characteristic 1D velocity variation scale for planar models with $n_{\mathrm{H}}=10^{6} \mathrm{~cm}^{-3}, v_{\text {bow }}=40 \mathrm{~km} \mathrm{~s}^{-1}$, and various values of $b_{\|}$. Only models with $T_{\max }>1000 \mathrm{~K}$ are displayed.

expansion terms versus the 1D compression in the 1D models remains internally self-consistent. We refer to this as the " $z$-axis" approximation.

In reality, the velocity, $v_{\perp \text {,post }}$, perpendicular to the shock surface of the post-shock gas decreases after the shock, while the velocity, $v_{\|}$, parallel to the shock surface remains constant. Thus, the post-shock streamlines are curved. For a planar shock, the position of the post-shock gas with respect to the entrance point at a time, $t$, is given by

$\boldsymbol{p}(t)=t v_{\|} \boldsymbol{u}_{\|}+d(t) \boldsymbol{u}_{\perp}=t v_{\text {bow }} \boldsymbol{u}_{z}+\left(d(t)-t v_{\perp}\right) \boldsymbol{u}_{\perp}$, 

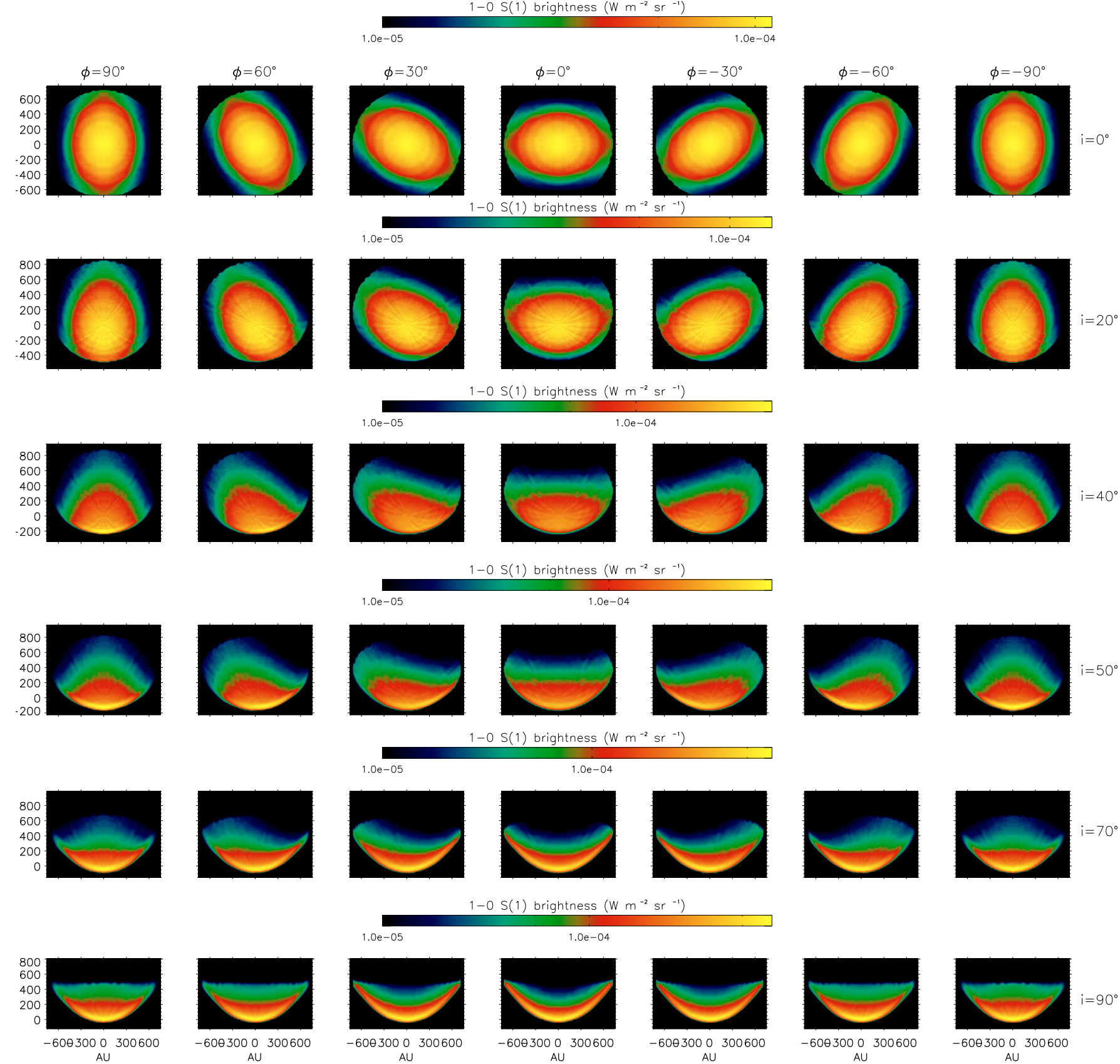

Fig. A.9. The standard shock with $\theta=0^{\circ}$ (magnetic field perpendicular to the bow axis) seen at different inclinations (top to bottom) and different azimuthal $\phi$-angles (left to right). The bow parameters are $n_{\mathrm{H}}=10^{6} \mathrm{~cm}^{3}, v_{\text {bow }}=40 \mathrm{~km} \mathrm{~s}^{-1}, b=5, \beta=2.0, r_{0}=1000$ AU. The colour scales are logarithmic.

where $d(t)$ is the distance travelled by the post-shock gas at time $t$ in the planar shock model. $\boldsymbol{u}_{\|}$and $\boldsymbol{u}_{\perp}$ are unit vectors parallel and perpendicular, respectively, to the shock surface; and we have used that

$v_{\text {bow }} \boldsymbol{u}_{z}=v_{\|} \boldsymbol{u}_{\|}+v_{\perp} \boldsymbol{u}_{\perp}$.

The radial offset of $\boldsymbol{p}(t)$ from the $z$-axis is then

$$
\begin{aligned}
r(t)=\boldsymbol{p}(t) \cdot \boldsymbol{u}_{r} & =\left(d(t)-t v_{\perp}\right) \boldsymbol{u}_{\perp} \cdot \boldsymbol{u}_{r} \\
& =\left(t v_{\perp}-d(t)\right) \cos (\omega)>0,
\end{aligned}
$$

where $\omega=\arctan (\mathrm{d} r / \mathrm{d} z)=\arctan \left(v_{\perp} / v_{\|}\right)$is the angle between the local tangent to the bow and the $z$-axis. Similarly, the exact position in the $z$-direction is

$z(t)=\boldsymbol{p}(t) \cdot \boldsymbol{u}_{z}=t v_{\text {bow }}+\left(d(t)-t v_{\perp}\right) \boldsymbol{u}_{\perp} \cdot \boldsymbol{u}_{z}$

$$
=t v_{\text {bow }}+\left(d(t)-t v_{\perp}\right) \sin (\omega) .
$$

We can estimate the accuracy of the $z$-axis approximation by looking at the radial displacement of the peak of the $\mathrm{H}_{2}$ emissivity from the $z$-axis, which is simply

$\Delta r_{\text {peak }}=r\left(t_{\text {peak }}\right)$,

and at the geometrical "distortion" it introduces in our bow images, which we measure as the angular displacement between the position of the peak of the $\mathrm{H}_{2}$ emissivity and the $z$-axis, given by

$\Delta \Omega=\arctan \left(r\left(t_{\text {peak }}\right) / z\left(t_{\text {peak }}\right)\right)$.

These two quantities are plotted in Figs. A.1 and A.2, respectively, as a function of $v_{\perp}$ for planar models along a bowshock 


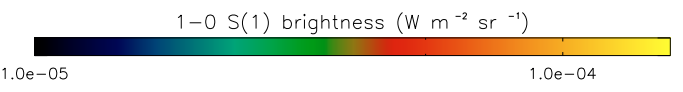

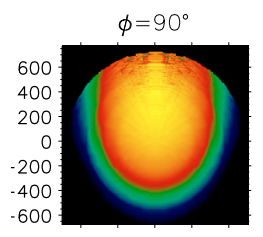
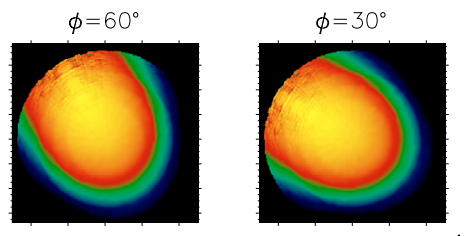

$\phi=0^{\circ}$

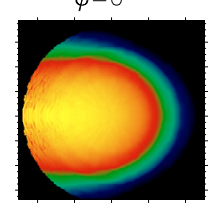

$\phi=-30^{\circ}$
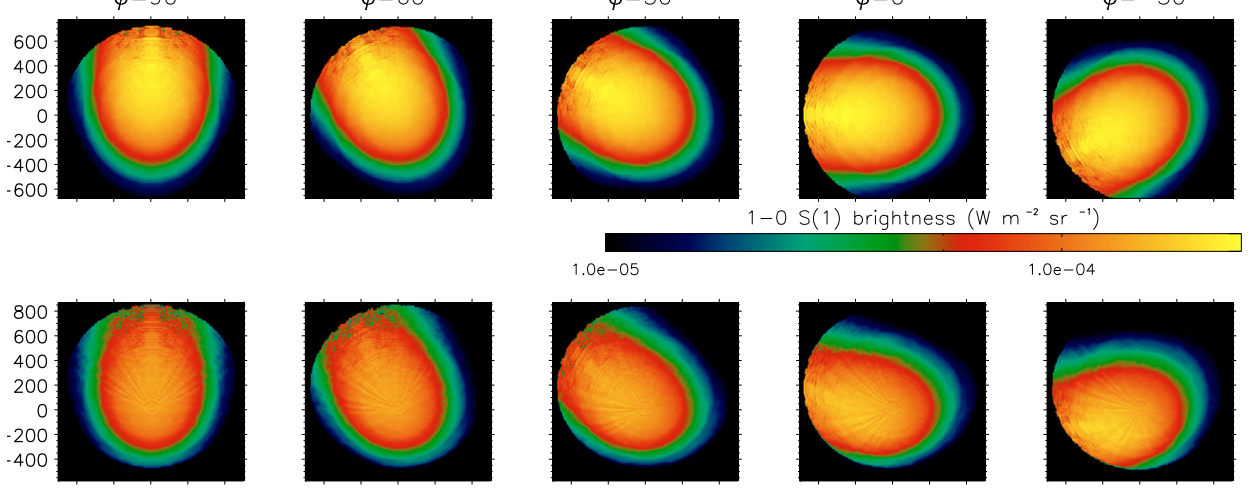

$-0 \mathrm{~S}(1)$ brightness $\left(\mathrm{W} \mathrm{m}^{-2} \mathrm{sr}^{-1}\right)$
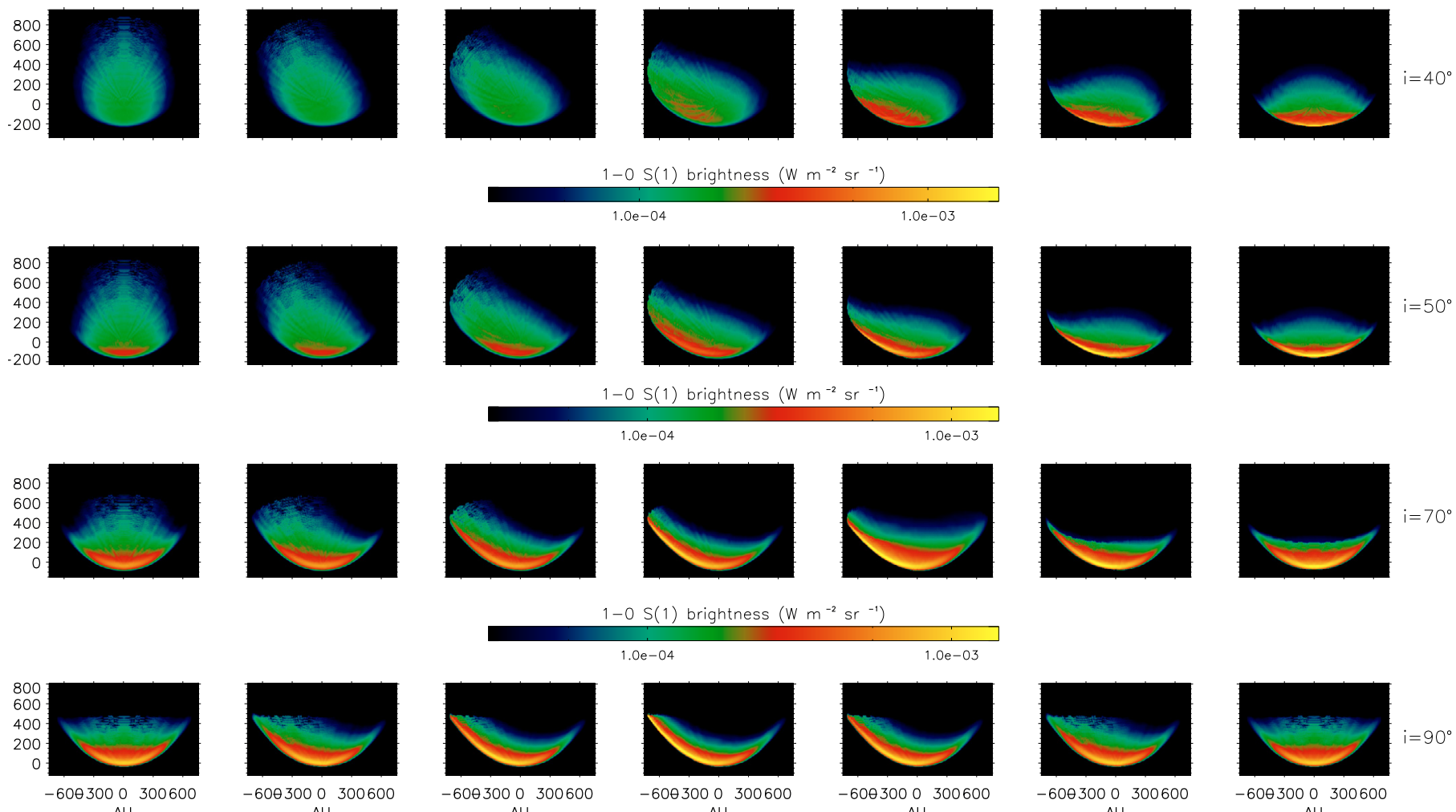

$\mathrm{AU}$

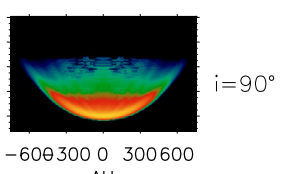

$\mathrm{AU}$

Fig. A.10. Same as Fig. A.9, but with $\theta=30^{\circ}$.

with $b_{\|}=5, v_{\text {bow }}=40 \mathrm{~km} \mathrm{~s}^{-1}$, and various preshock densities. Note that $v_{\perp}$ is maximum at the apex and decreases along the bow wings, until the temperature is too low to emit significantly in rovibrational $\mathrm{H}_{2}$ lines $(T<1000 \mathrm{~K})$.

The radial displacement $\Delta r_{\text {peak }}$ (Fig. A.1) is highly dependent on the pre-shock density since its value depends on the cooling distance. However, the maximum value is generally smaller than the pixel size used in the models displayed in this paper. The effect of using the $z$-axis approximation is thus insignificant. The biggest effect is found in the right hand side of Fig. 2 in the model with $n_{\mathrm{H}}=10^{4} \mathrm{~cm}^{-3}$. The pixel size is here $41.5 \mathrm{AU}$, which means that by using the $z$-axis approximation we misplace the peak $\mathrm{H}_{2}$ emissivity 4 pixels in the $r$-direction. Even in this case, however, the distortion introduced in the map morphology is very small, because the angular displacement from the vertical, $\Delta \Omega$ (Fig. A.2) remains low at $<5^{\circ}$ all along the bow shock, for all values of preshock densities.

The effect of a lower $b_{\|}$on $\Delta r_{\text {peak }}$ and $\Delta \Omega$ is illustrated in Figs. A. 3 and A.4 respectively, in the case $n_{\mathrm{H}}=10^{6} \mathrm{~cm}^{-3}$. Since the value of the radial displacement depends on the distance travelled by the post-shock gas, it decreases in shocks with a lower $b_{\|}$, where the cooling distance is shorter. At the same time, the distortion angle $\Delta \Omega$ of the peak emission region from the vertical is virtually unchanged and remains small $\sim 5^{\circ}$ for $b_{\|}>1$. In the case where $b_{\|}=1$, the shock becomes J-type when $v_{\perp}>33 \mathrm{~km} \mathrm{~s}^{-1}$ due to $\mathrm{H}_{2}$ dissociation. The bulk of rovibrational $\mathrm{H}_{2}$ emission is then emitted at much lower velocities and higher compression than in C-shocks, and the angular deviation increases dramatically to $40^{\circ}$. However at the same time 


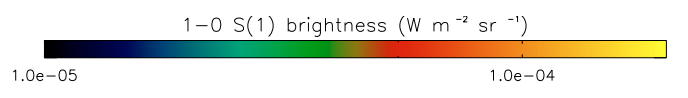

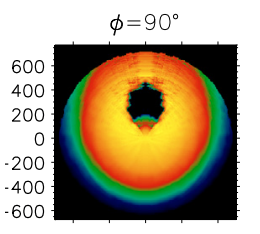
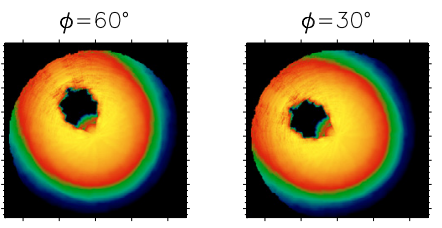

$\phi=0^{\circ}$

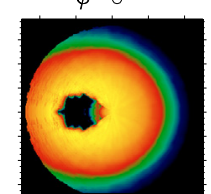

$\phi=-30^{\circ}$

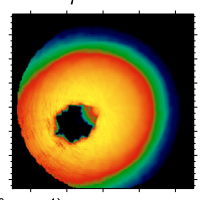

$-0 \mathrm{~S}(1)$ brightness $\left(\mathrm{W} \mathrm{m}^{-2} \mathrm{sr}^{-1}\right)$
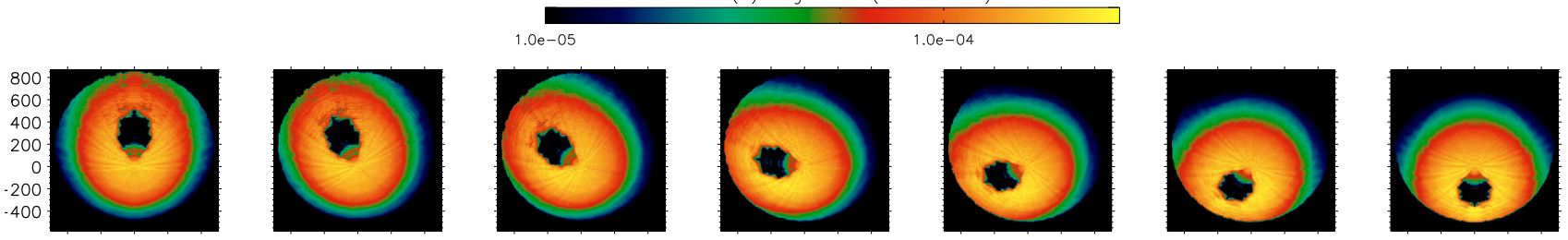

$1-0 \mathrm{~S}(1)$ brightness $\left(\mathrm{W} \mathrm{m}^{-2} \mathrm{sr}^{-1}\right)$
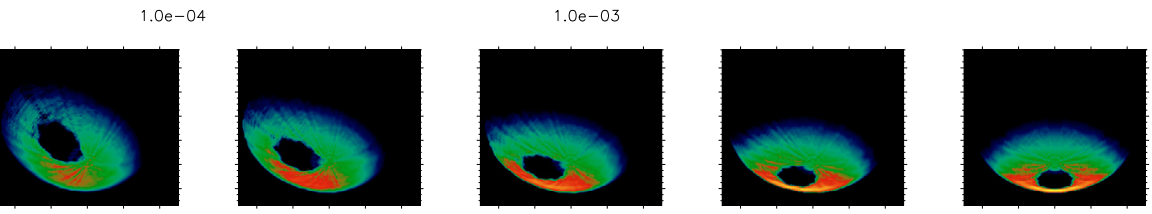

$1-0 \mathrm{~S}(1)$ brightness $\left(\mathrm{W} \mathrm{m}^{-2} \mathrm{sr}^{-1}\right)$
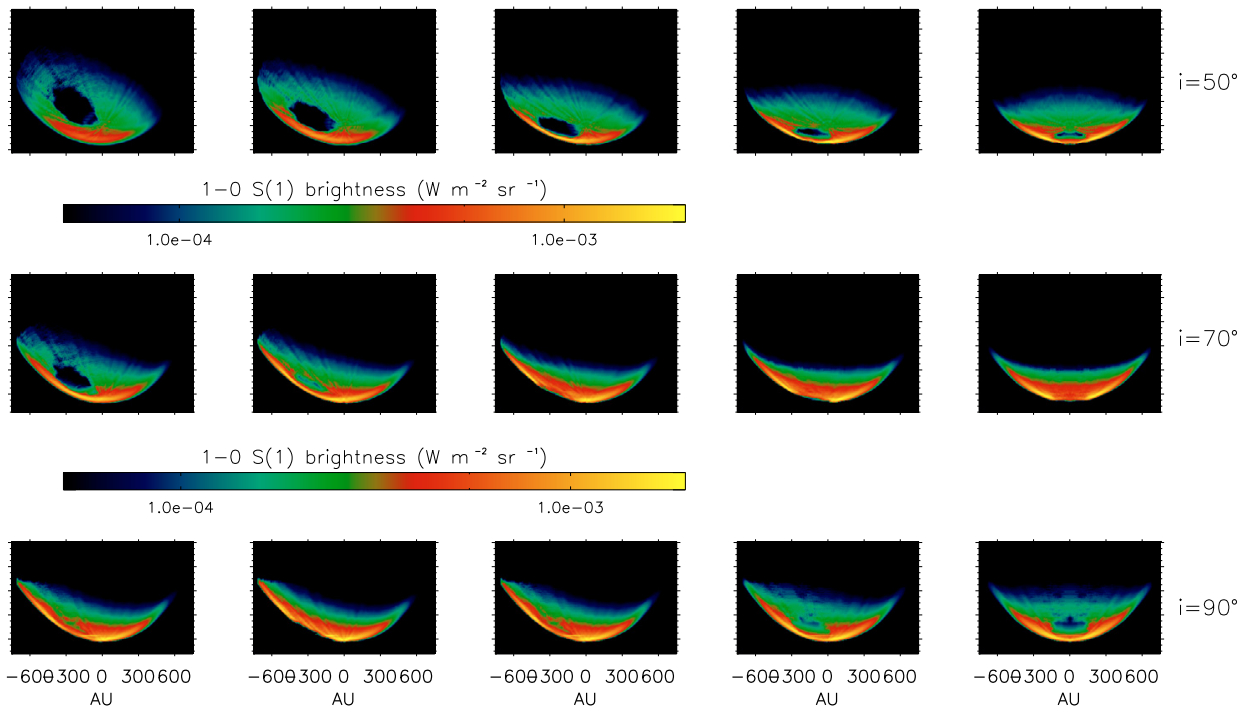

Fig. A.11. Same as Fig. A.9, but with $\theta=60^{\circ}$.

the cooling distance also shrinks dramatically, so that the offset from the vertical is completely negligible.

We conclude that the z-axis approximation does not introduce a significant distortion of the bow emission morphology for the parameter range and map resolution explored in this paper.

\section{A.2. Stretching along the z-axis}

In building our 3D model, we also assumed that the position along the $z$-axis streamline is stretched uniformly to reach a full cooling distance of $\left(d_{\perp, \text { cool }}^{2}+d_{\|, \text {cool }}^{2}\right)^{1 / 2}$, where $d_{\perp \text {, cool }}$ and $d_{\|, \text {cool }}=t_{\text {cool }} v_{\|}$are the distance travelled by the post-shock gas at time $t_{\text {cool }}$ (where $T=1000 \mathrm{~K}$ ) perpendicular and parallel to the shock surface, respectively. The rationale behind is that the local velocity gradient $\mathrm{d} V_{z}(t) / \mathrm{d} z$ remains unchanged from the
1D case, consistent with our use of the 1D temperature and emissivity distribution.

In other words, we use the following expression for the approximate position of the post-shock gas along the $z$-axis:

$z_{\text {approx }}(t)=d(t) / \sin (\delta)$,

where the "stretching factor" $1 / \sin \delta$ is defined as

$\sin (\delta)=\frac{d_{\perp, \mathrm{cool}}}{\sqrt{d_{\perp, \mathrm{cool}}^{2}+d_{\|, \mathrm{cool}}^{2}}}=\frac{d_{\perp, \mathrm{cool}}}{\sqrt{d_{\perp, \mathrm{cool}}^{2}+v_{\|}^{2} t_{\mathrm{cool}}^{2}}}$

In order to estimate the accuracy of this approximation we look at the exact position $z(t)$ of the peak $\mathrm{H}_{2}$ emissivity along the $z$-axis, given in Eq. (A.6), and calculate the relative error

$\frac{\Delta z_{\text {peak }}}{z_{\text {peak }}}=\frac{z_{\text {approx }}\left(t_{\text {peak }}\right)-z\left(t_{\text {peak }}\right)}{z_{\text {approx }}\left(t_{\text {peak }}\right)}$ 


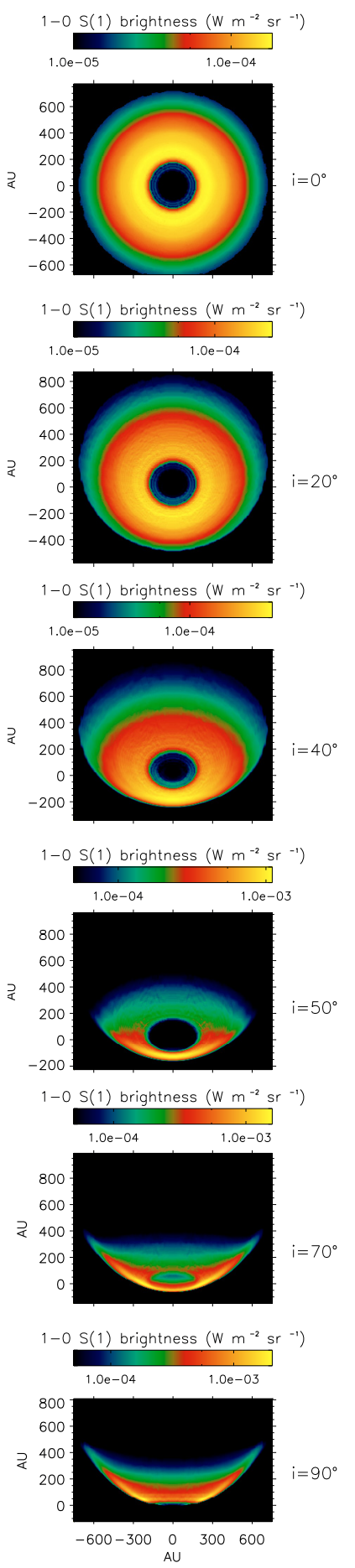

Fig. A.12. Same as Fig. A.9, but with $\theta=90^{\circ}$. A single column is shown since the result does not depend on $\phi$ (the magnetic field is parallel to the bow axis and the shock is thus axisymmetric).

This quantity is displayed in (Fig. A.5) for various preshock densities and $b_{\|}=5$. The relative error in $z_{\text {peak }}$ first increases as we move away from the bow apex (decreasing $v_{\perp}$ ), and reaches a maximum value, before it decreases as we move further along the wing. The error never exceeds $15 \%$ and the approximation gets better at lower densities.

The same graph in shown in (Fig. A.6) for lower values of $b_{\|}$and a pre-shock density $n_{\mathrm{H}}=10^{6} \mathrm{~cm}^{-3}$. The relative error on $z$ tends to increase at lower b-field, but it does not exceed $35 \%$ in $\mathrm{C}$-shocks. This is a rather high value but corresponds to
$<3$ pixels in our standard model. Furthermore, as with $\Delta\left(r_{\text {peak }}\right)$, the absolute error $\Delta\left(z_{\text {peak }}\right)$ in AUs actually decreases at lower $b$ and would not be resolvable with current instruments (this is even more true for the J-shocks). Therefore this approximation also seems acceptable for our range of parameters.

\section{A.3. Minimum scale of curvature}

When using the output of $1 \mathrm{D}$ shock models, we are ignoring the effect of the bow geometry on the velocity, density, and temperature of the post-shock emitting material. Several effects will be present: first, since the streamline is not exactly vertical, the emitting material is moving in cones rather than cylinders and thus will suffer some adiabatic expansion. However we have seen that the angle is very small in the region of rovibrational $\mathrm{H}_{2}$ emission (a few degrees, see Figs. A.2 and A.4) therefore this effect can be neglected. Second, the bow curvature makes the shock speed and postshock pressure vary along the shock surface, introducing a transverse thermal and magnetic pressure gradient across streamlines. Calculating such a complex effect is outside the scope of the present paper. However we expect that it would remain small with respect to $1 \mathrm{D}$ shock compression if the local radius of curvature of the bow (determining these transverse gradients) is much larger than the characteristic scale of velocity (i.e. pressure) variation in the $\mathrm{H}_{2}$ emission zone of the 1D shock, given by

$r_{v}=\left(v_{\text {peak }}-v_{\perp}\right) /\left(\mathrm{d} V_{n} / \mathrm{d} s\right)\left(t_{\text {peak }}\right)$,

where $\left(\mathrm{d} V_{n} / \mathrm{d} s\right)$ is the velocity gradient in the 1D shock model at the peak of $\mathrm{H}_{2}$ line emissivity.

This characteristic scale is plotted along a bow of speed $40 \mathrm{~km} \mathrm{~s}^{-1}$ for various preshock densities in Fig. A.7, and for various $b_{\|}$in Fig. A.8. Like the cooling distance, the scale varies almost inversely with $n_{\mathrm{H}}$, dropping from $3000 \mathrm{AU}$ to $60 \mathrm{AU}$ for $n_{\mathrm{H}}=10^{4}-10^{6} \mathrm{~cm}^{-3}$ when $b_{\|}=5$, and decreasing in proportion to $b_{\|}$for a fixed density (when the shock is C-type). All the bowshock models presented in this paper (in particular those fitted to the OMC1 bowshock) have values of $r_{0}$ that exceed this characteristic scale for the relevant $n_{\mathrm{H}}$, suggesting that the $1 \mathrm{D}$ approximation may be approximately valid, especially in the bow wings.

\section{Appendix B: Effect of magnetic field direction and viewing angle}

Here we show in detail how the magnetic field direction and the inclination to the line of sight affect the morphology of the projected emission of our standard bow shock model. The standard model has the following input parameters: $n_{\mathrm{H}}=10^{6} \mathrm{~cm}^{3}$, $v_{\text {bow }}=40 \mathrm{~km} \mathrm{~s}^{-1}, b=5, \beta=2.0, r_{0}=1000$ AU. Figure A.9 displays the effect of changing the "rotation" angle $\phi$ from $+90^{\circ}$ to $-90^{\circ}$ (left to right) and the inclination $i$ from 0 to $+90^{\circ}$ (top to bottom) when $\theta=0^{\circ}$ (magnetic field perpendicular to the bow axis). Figure A.10 shows the same for $\theta=30^{\circ}$ and Fig. A.11 the same for $\theta=60^{\circ}$. The case of $\theta=90^{\circ}$ is illustrated in Fig. A.12, which contains a single column since the result does not depend on $\phi$ (magnetic field parallel to the bow axis). Note that maps for $\phi>90^{\circ}$ can be deduced from the map with $\phi^{\prime}=180^{\circ}-\phi$ by applying mirror symmetry with respect to $x=0$. Similarly, maps for $\phi<-90^{\circ}$ can be deduced from the map with $\phi^{\prime}=-\left(180^{\circ}+\phi\right)$. 


\section{References}

Allen, D. A., \& Burton, M. G. 1993, Nature, 363, 54

Axon, D. J., \& Taylor, K. 1984, MNRAS, 207, 241

Baek, C. H., Kim, J., \& Choi, M. 2009, ApJ, 690, 944

Chrysostomou, A., Hough, J. H., Burton, M. G., \& Tamura, M. 1994, MNRAS, 268, 325

Colgan, S. W. J., Schultz, A. S. B., Kaufman, M. J., Erickson, E. F., \& Hollenbach, D. J. 2007, ApJ, 671, 536

Crutcher, R. M., Troland, T. H., Lazareff, B., Paubert, G., \& Kazès, I. 1999, ApJ, 514, L121

Cunningham, N. 2006, Ph.D. Thesis, University of Colorado

Davis, C. J., Froebrich, D., Stanke, T., et al. 2009, A\&A, 496, 153

Draine, B. T. 1980, ApJ, 241, 1021

Draine, B. T., \& McKee, C. F. 1993, ARA\&A, 31, 373

Eislöffel, J., Mundt, R., \& Bohm, K.-H. 1994, AJ, 108, 1042

Flower, D. R., \& Pineau des Forêts, G. 2003, MNRAS, 343, 390

Genzel, R., Reid, M. J., Moran, J. M., \& Downes, D. 1981, ApJ, 244, 884

Greenhill, L. J., Gwinn, C. R., Schwartz, C., Moran, J. M., \& Diamond, P. J. 1998, Nature, 396, 650

Gustafsson, M. 2006, Ph.D. Thesis, University of Aarhus

Gustafsson, M., Kristensen, L. E., Clénet, Y., et al. 2003, A\&A, 411, 437

Hough, J. H., Axon, D. J., Burton, M. G., et al. 1986, MNRAS, 222, 629

Kristensen, L. E., Ravkilde, T. L., Field, D., Lemaire, J. L., \& Pineau Des Forêts, G. 2007, A\&A, 469, 561

Kristensen, L. E., Ravkilde, T. L., Pineau Des Forêts, G., et al. 2008, A\&A, 477, 203

Lacombe, F., Gendron, E., Rouan, D., et al. 2004, A\&A, 417, L5
Le Bourlot, J., Pineau des Forêts, G., Flower, D. R., \& Cabrit, S. 2002, MNRAS, 332,985

Menten, K. M., Reid, M. J., Forbrich, J., \& Brunthaler, A. 2007, A\&A, 474, 515

Nissen, H. D., Gustafsson, M., Lemaire, J. L., et al. 2007, A\&A, 466, 949

Norris, R. P. 1984, MNRAS, 207, 127

O'Connell, B., Smith, M. D., Davis, C. J., et al. 2004, A\&A, 419, 975

O'Connell, B., Smith, M. D., Froebrich, D., Davis, C. J., \& Eislöffel, J. 2005, A\&A, 431, 223

Raga, A. C., de Gouveia Dal Pino, E. M., Noriega-Crespo, A., Mininni, P. D., \& Velázquez, P. F. 2002, A\&A, 392, 267

Raga, A. C., Esquivel, A., Riera, A., \& Velázquez, P. F. 2007, ApJ, 668, 310

Ravkilde, T. L. 2007, Master's thesis, Department of Physics and Astronomy, University of Aarhus, Ny Munkegade, Bld. 1520, DK-8000 Aarhus C, Denmark, available online at http://www. phys.au.dk/ ravkilde/msc/ Reipurth, B., \& Bally, J. 2001, ARA\&A, 39, 403

Schultz, A. S. B., Colgan, S. W. J., Erickson, E. F., et al. 1999, ApJ, 511, 282

Simpson, J. P., Colgan, S. W. J., Erickson, E. F., Burton, M. G., \& Schultz, A. S. B. 2006, ApJ, 642, 339

Smith, M. D. 1991, MNRAS, 252, 378

Smith, M. D. 1992, ApJ, 390, 447

Smith, M. D., Khanzadyan, T., \& Davis, C. J. 2003, MNRAS, 339, 524

Smith, M. D., O’Connell, B., \& Davis, C. J. 2007, A\&A, 466, 565

Stolovy, S. R., Burton, M. G., Erickson, E. F., et al. 1998, ApJ, 492, L151

Suttner, G., Smith, M. D., Yorke, H. W., \& Zinnecker, H. 1997, A\&A, 318, 595

Takami, M., Chrysostomou, A., Ray, T. P., et al. 2006, ApJ, 641, 357

Takami, M., Beck, T. L., Pyo, T.-S., McGregor, P., \& Davis, C. 2007, ApJ, 670, L33

Tamura, M., Kandori, R., Kusakabe, N., et al. 2006, ApJ, 649, L29 